\title{
Parton distributions from nonlocal chiral SU(3) effective theory: Flavor asymmetries
}

\author{
Y. Salamu $\odot,{ }^{1,2}$ Chueng-Ryong Ji® ${ }^{3}$ W. Melnitchouk $\odot,{ }^{4}$ A. W. Thomas $\odot,{ }^{5}$ P. Wang $\odot,{ }^{1,6}$ and X. G. Wang ${ }^{5}$ \\ ${ }^{1}$ Institute of High Energy Physics, CAS, Beijing 100049, China \\ ${ }^{2}$ School of Physical Sciences, University of Chinese Academy of Sciences, Beijing 100049, China \\ ${ }^{3}$ Department of Physics, North Carolina State University, Raleigh, North Carolina 27695, USA \\ ${ }^{4}$ Jefferson Lab, Newport News, Virginia 23606, USA \\ ${ }^{5}$ CoEPP and CSSM, Department of Physics, University of Adelaide, Adelaide SA 5005, Australia \\ ${ }^{6}$ Theoretical Physics Center for Science Facilities, CAS, Beijing 100049, China
}

(Received 19 July 2019; published 21 November 2019)

\begin{abstract}
Using recently derived results for one-loop hadronic splitting functions from a nonlocal implementation of chiral effective theory, we study the contributions from pseudoscalar meson loops to flavor asymmetries in the proton. Constraining the parameters of the regulating functions by inclusive production of $n, \Delta^{++}, \Lambda$, and $\Sigma^{*+}$ baryons in $p p$ collisions, we compute the shape of the light antiquark asymmetry $\bar{d}-\bar{u}$ in the proton and the strange asymmetry $s-\bar{s}$ in the nucleon sea. With these constraints, the magnitude of the $\bar{d}-\bar{u}$ asymmetry is found to be compatible with that extracted from the Fermilab E866 Drell-Yan measurement, with no indication of a sign change at large values of $x$, and an integrated value in the range $\langle\bar{d}-\bar{u}\rangle \approx 0.09-0.17$. The $s-\bar{s}$ asymmetry is predicted to be positive at $x>0$, with compensating negative contributions at $x=0$, and an integrated $x$-weighted moment in the range $\langle x(s-\bar{s})\rangle \approx(0.9-2.5) \times 10^{-3}$.
\end{abstract}

DOI: 10.1103/PhysRevD.100.094026

\section{INTRODUCTION}

It is well known that a complete characterization of nucleon substructure must go beyond three valence quarks. One of the great challenges of modern hadron physics is to unravel the precise role of hidden flavors in the structure of the nucleon. The observation of the $\bar{d}-\bar{u}$ flavor asymmetry in the light quark sea of the proton [1-4], following its prediction by Thomas a decade earlier [5] on the basis of chiral symmetry breaking [6,7], has been one of the seminal results in hadronic physics over the past two decades. It has led to a major reevaluation of our understanding of the role of the nonvalence components of the nucleon and their origin in QCD [8-10].

The role that strange quarks, in particular, play in the nucleon has also been the focus of attention in hadronic physics for many years. Early polarized deep-inelastic scattering (DIS) experiments suggested that a surprisingly large fraction of the proton's spin might be carried by strange quarks [11], in contrast to the naive quark model expectations [12]. One of the guiding principles for

Published by the American Physical Society under the terms of the Creative Commons Attribution 4.0 International license. Further distribution of this work must maintain attribution to the author(s) and the published article's title, journal citation, and DOI. Funded by SCOAP ${ }^{3}$. understanding the nonperturbative features of strange quarks and antiquarks in the nucleon sea has been chiral symmetry breaking in QCD. While the generation of $s \bar{s}$ pairs through perturbative gluon radiation typically produces symmetric $s$ and $\bar{s}$ distributions (at least up to two loop corrections [13]), any significant difference between the momentum dependence of the $s$ and $\bar{s}$ distributions would be a clear signal of nonperturbative effects [14-19].

In the previous paper [20], we presented the proton $\rightarrow$ pseudoscalar meson $(\phi)+$ baryon splitting functions for the intermediate octet $(B)$ and decuplet $(T)$ baryon configurations in nonlocal chiral effective theory [21,22]. From the calculated splitting functions, the parton distribution functions (PDFs) of the nucleon are obtained as convolutions of these with PDFs of the intermediate state mesons and baryons [23-25]. Here we apply the results from [20] to compute, for the first time within the nonlocal theory, sea quark PDF asymmetries in the proton, including the light antiquark flavor asymmetry $\bar{d}-\bar{u}$ and the strange quark asymmetry $s-\bar{s}$. Using SU(3) relations for the intermediate state hadron PDFs, the only free parameters in the calculation of the asymmetries are the mass parameters appearing in the ultraviolet regulator functions. These will be determined by fitting cross section data from inclusive baryon production in high energy $p p$ scattering, using the same splitting functions that appear in the PDF asymmetries. All other parameters, including coupling constants and intermediate state baryon and meson PDFs, are fixed. 
We begin in Sec. II by summarizing the convolution formulas for the quark and antiquark PDFs in terms of the fluctuations of the nucleon into its meson-baryon lightcone components. The calculation of the PDFs of the intermediate state baryons and mesons in the chiral theory is discussed in detail in Sec. III. Numerical results for the sea quark asymmetries are presented in Sec. IV, where we compare the results for $\bar{d}-\bar{u}$ with those extracted from Drell-Yan and semi-inclusive DIS measurements, and compare predictions for $s-\bar{s}$ asymmetries with some recent PDF parametrizations. Finally, in Sec. V we summarize our results and discuss future measurements which could further constrain the PDF asymmetries experimentally.

\section{CONVOLUTION FORMULAS}

Using the crossing symmetry properties of the spinaveraged PDFs, $q(-x)=-\bar{q}(x)$, the $n$th Mellin moment $(n \geq 1)$ of the distribution for a given flavor $q$ $(q=u, d, s, \ldots)$ is defined by

$$
Q^{(n-1)}=\int_{0}^{1} d x x^{n-1}\left[q(x)+(-1)^{n} \bar{q}(x)\right] .
$$

In the operator product expansion, the moments $Q^{(n-1)}$ are related to matrix elements of local twist-two, spin- $n$ operators $\mathcal{O}_{q}^{\mu_{1} \cdots \mu_{n}}$ between nucleon states with momentum $p$,

$$
\left\langle N(p)\left|\mathcal{O}_{q}^{\mu_{1} \cdots \mu_{n}}\right| N(p)\right\rangle=2 Q^{(n-1)} p^{\mu_{1}} \cdots p^{\mu_{n}},
$$

where the operators are given by

$$
\left.\mathcal{O}_{q}^{\mu_{1} \cdots \mu_{n}}=i^{n-1} \bar{q} \gamma^{\left\{\mu_{1}\right.} \stackrel{\leftrightarrow}{D} \mu_{2} \ldots \stackrel{\leftrightarrow}{D}^{\mu_{n}}\right\} q
$$

with $\stackrel{\leftrightarrow}{D}=\frac{1}{2}(\vec{D}-\overleftarrow{D})$, and the braces $\{\cdots\}$ denote symmetrization of Lorentz indices. The effective theory allows the quark operators $\mathcal{O}_{q}$ to be matched to hadronic operators $\mathcal{O}_{j}$ with the same quantum numbers [23],

$$
\mathcal{O}_{q}^{\mu_{1} \cdots \mu_{n}}=\sum_{j} c_{q / j}^{(n)} \mathcal{O}_{j}^{\mu_{1} \cdots \mu_{n}}
$$

where the coefficients $c_{q / j}^{(n)}$ are the $n$th moments of the PDF $q_{j}(x)$ in the hadronic configuration $j$,

$$
c_{q / j}^{(n)}=\int_{-1}^{1} d x x^{n-1} q_{j}(x) \equiv Q_{j}^{(n-1)} .
$$

The nucleon matrix elements of the hadronic operators $\mathcal{O}_{j}^{\mu_{1} \cdots \mu_{n}}$ are given in terms of moments of the splitting functions $f_{j}(y)$,

$$
\left\langle N(p)\left|\mathcal{O}_{j}^{\mu_{1} \cdots \mu_{n}}\right| N(p)\right\rangle=2 f_{j}^{(n)} p^{\left\{\mu_{1}\right.} \cdots p^{\left.\mu_{n}\right\}},
$$

where

$$
f_{j}^{(n)}=\int_{-1}^{1} d y y^{n-1} f_{j}(y)
$$

with $y$ the light-cone momentum fraction of the nucleon carried by the hadronic state $j$. The operator relation in Eq. (4) then gives rise to the convolution formula for the PDFs [23,24],

$$
\begin{aligned}
q(x) & =\sum_{j}\left[f_{j} \otimes q_{j}^{v}\right](x) \\
& \equiv \sum_{j} \int_{0}^{1} d y \int_{0}^{1} d z \delta(x-y z) f_{j}(y) q_{j}^{v}(z),
\end{aligned}
$$

where $q_{j}^{v} \equiv q_{j}-\bar{q}_{j}$ is the valence distribution for the quark flavor $q$ in the hadron $j$. The complete set of splitting functions $f_{j}(y)$ for octet and decuplet baryons is given in Ref. [20].

In the present analysis we work under the basic assumption that the bare baryon states are composed of three valence quarks plus quark-antiquark pairs that are generated perturbatively through gluon radiation. Such contributions will effectively cancel in any differences of PDFs, such as $\bar{d}-\bar{u}$ or $s-\bar{s}$. We therefore focus only on the nonperturbative contributions to sea quark PDFs which arise from pseudoscalar meson loops. In this approximation antiquark distributions arise only from diagrams involving direct coupling to mesons, as in the meson rainbow and bubble diagrams in Fig. 1. The meson loop contribution to the antiquark PDFs in the nucleon can then be written as

$$
\bar{q}(x)=\sum_{B, T, \phi}\left[\left(f_{\phi B}^{(\mathrm{rbw})}+f_{\phi T}^{(\mathrm{rbw})}+f_{\phi}^{(\mathrm{bub})}\right) \otimes \bar{q}_{\phi}\right](x),
$$

where $f_{\phi B}^{(\mathrm{rbw})}$ and $f_{\phi T}^{(\mathrm{rbw})}$ represent splitting functions from the rainbow diagrams with octet and decuplet baryons in Figs. 1(a) and 1(b), respectively, $f_{\phi}^{(\text {bub })}$ is the splitting function for the meson bubble diagram in Fig. 1(c), and $\bar{q}_{\phi}(x)$ is the antiquark PDF in the meson.

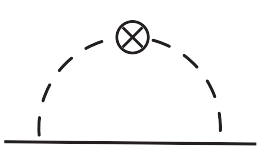

(a)

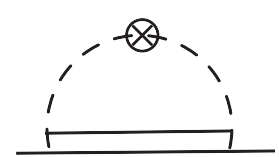

(b)

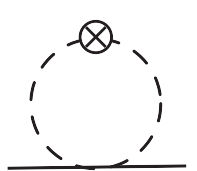

(c)
FIG. 1. One-meson loop diagrams contributing to quark and antiquark PDFs in the nucleon, representing (a) the rainbow diagram with octet baryon (solid lines) intermediate state; (b) the rainbow diagram with decuplet baryon (double solid lines) intermediate state; and (c) the meson (dashed lines) bubble diagram. The symbol " $\otimes$ " represents an operator insertion. 


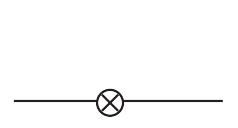

(a)

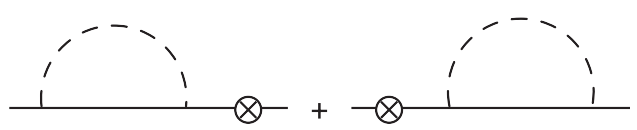

(b)

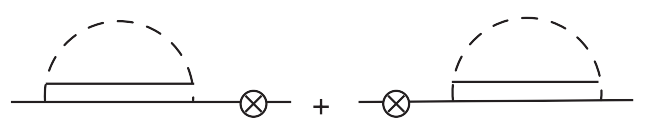

(c)

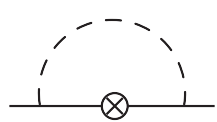

(d)

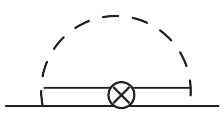

(g)

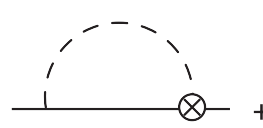

(e)

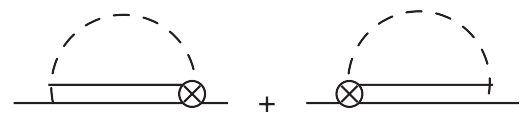

(h)

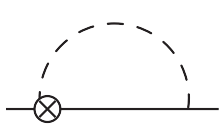

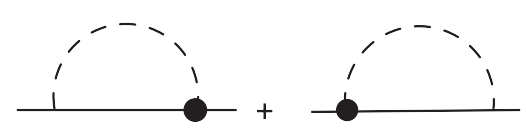

(f)

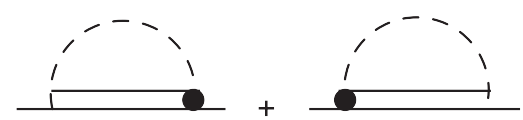

(i)

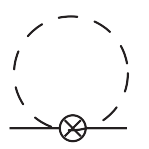

(j)

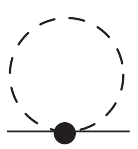

(k)

FIG. 2. Contributions to quark PDFs in the nucleon from baryon coupling diagrams, representing (a) coupling to the bare nucleon; (b), (c) contributions from wave function renormalization with octet and decuplet baryon intermediate states; (d) the rainbow diagram with octet baryon; (e),(f) Kroll-Ruderman and gauge link (filled circle) Kroll-Ruderman diagrams with octet baryon; (g) the rainbow diagram with decuplet baryon; (h),(i) Kroll-Ruderman diagrams with decuplet baryon; and (j),(k) meson tadpole and gauge link tadpole diagrams.

Contributions to quark PDFs can in principle come from both meson coupling and baryon coupling diagrams. The latter are illustrated in Fig. 2 and involve the bare nucleon coupling [Fig. 2(a)], wave function renormalization [Figs. 2(b) and 2(c), with octet and decuplet baryon intermediate states, respectively], baryon rainbow [Figs. 2(d) and 2(g)], Kroll-Ruderman (KR) [Figs. 2(e) and 2(h)], and meson tadpole [Fig. 2(j)] diagrams, along with gauge link dependent Kroll-Ruderman [Figs. 2(f) and 2(i)] and tadpole [Fig. 2(k)] diagrams. Within the valence approximation, all of these diagrams will contribute to the $u$ and $d$ quarks in the nucleon. However, for the strange quark the bare coupling and wave function renormalization diagrams do not contribute. The total nonperturbative contribution from meson loops to the quark PDF in the nucleon can then be written

$$
\begin{aligned}
q(x)= & Z_{2} q^{(0)}(x)+\sum_{B, T, \phi}\left\{\left[\left(f_{\phi B}^{(\mathrm{rbw})}+f_{\phi T}^{(\mathrm{rbw})}+f_{\phi}^{(\mathrm{bub})}\right) \otimes q_{\phi}\right](x)\right. \\
& +\left[\bar{f}_{B \phi}^{(\mathrm{rbw})} \otimes q_{B}\right](x)+\left[\bar{f}_{B}^{(\mathrm{KR})} \otimes q_{B}^{(\mathrm{KR})}\right](x)+\left[\delta \bar{f}_{B}^{(\mathrm{KR})} \otimes q_{B}^{(\delta)}\right](x) \\
& +\left[\bar{f}_{T \phi}^{(\mathrm{rbw})} \otimes q_{T}\right](x)+\left[\bar{f}_{T}^{(\mathrm{KR})} \otimes q_{T}^{(\mathrm{KR})}\right](x)+\left[\delta \bar{f}_{T}^{\mathrm{KR})} \otimes q_{T}^{(\delta)}\right](x) \\
& \left.+\left[\bar{f}_{\phi}^{(\mathrm{tad})} \otimes q_{\phi}^{(\mathrm{tad})}\right](x)+\left[\delta \bar{f}_{\phi}^{(\mathrm{tad})} \otimes q_{\phi}^{(\delta)}\right](x)\right\},
\end{aligned}
$$

where $q^{(0)}$ is the quark PDF in the bare nucleon, and the wave function renormalization $Z_{2}$ arises from the summation over the diagrams in Figs. 2(a)-2(c) [26]. Following Ref. [24], we will work in terms of the same momentum fraction $y$ for all meson and baryon coupling diagrams in Figs. 1 and 2. Using the same definition of the convolution integral as in Eq. (8), it will be convenient therefore to define for each of the splitting functions in Eq. (10) involving the coupling to baryons the shorthand notation $\bar{f}_{j}(y) \equiv f_{j}(1-y)$ (see Sec. IV B below). Explicit expressions for the splitting functions $f_{B \phi}^{(\mathrm{rbw})}, f_{B}^{(\mathrm{KR})}$, $\delta f_{B}^{(\mathrm{KR})}, f_{T \phi}^{(\mathrm{rbw})}, f_{T}^{(\mathrm{KR})}, \delta f_{T}^{(\mathrm{KR})}, f_{\phi}^{(\mathrm{tad})}$, and $\delta f_{\phi}^{(\mathrm{tad})}$, which represent the diagrams in Figs. 2(d)-2(k), respectively, are given in Ref. [20]. The corresponding quark PDFs for the intermediate state octet and decuplet baryons are discussed in the next section.

\section{BARE BARYON AND MESON PDFS}

To calculate the contributions to the quark and antiquark distributions in the proton in the convolution formulas (9) 
and (10) requires the proton $\rightarrow$ baryon + meson splitting functions and the PDFs of the baryons and mesons to which the current couples. The full set of splitting functions was presented in our previous paper, Ref. [20]. In this section we derive the (valence) PDFs of the bare baryon and meson intermediate states using the same chiral SU(3) effective field theory framework that was used to compute the splitting functions.

\section{A. Operators and moments}

In the effective theory the quark level operators are matched to a sum of hadronic level operators whose matrix elements [see Eq. (4)] are given by the moments of the splitting functions, as in Eq. (6). Identifying all possible contributions from octet and decuplet baryon intermediate states that transform as vectors, the most general expression for the quark vector operator $\mathcal{O}_{q}^{\mu_{1} \cdots \mu_{n}}$ is given by [24,27]

$$
\begin{aligned}
& \mathcal{O}_{q}^{\mu_{1} \cdots \mu_{n}}=a^{(n)} i^{n} \frac{f_{\phi}^{2}}{4}\left\{\operatorname{Tr}\left[U^{\dagger} \lambda^{q} \partial_{\mu_{1}} \cdots \partial_{\mu_{n}} U\right]+\operatorname{Tr}\left[U \lambda^{q} \partial_{\mu_{1}} \cdots \partial_{\mu_{n}} U^{\dagger}\right]\right\} \\
& +\left[\alpha^{(n)}\left(\overline{\mathcal{B}} \gamma^{\mu_{1}} \mathcal{B} \lambda_{+}^{q}\right)+\beta^{(n)}\left(\overline{\mathcal{B}} \gamma^{\mu_{1}} \lambda_{+}^{q} \mathcal{B}\right)+\sigma^{(n)}\left(\overline{\mathcal{B}} \gamma^{\mu_{1}} \mathcal{B}\right) \operatorname{Tr}\left[\lambda_{+}^{q}\right]\right] p^{\mu_{2}} \ldots p^{\mu_{n}} \\
& +\left[\bar{\alpha}^{(n)}\left(\overline{\mathcal{B}} \gamma^{\mu_{1}} \gamma_{5} \mathcal{B} \lambda^{q}\right)+\bar{\beta}^{(n)}\left(\overline{\mathcal{B}} \gamma^{\mu_{1}} \gamma_{5} \lambda \underline{q} \mathcal{B}\right)+\bar{\sigma}^{(n)}\left(\overline{\mathcal{B}} \gamma^{\mu_{1}} \gamma_{5} \mathcal{B}\right) \operatorname{Tr}[\lambda \underline{q}]\right] p^{\mu_{2}} \cdots p^{\mu_{n}} \\
& +\left[\theta^{(n)}\left(\overline{\mathcal{T}}_{\alpha} \gamma^{\alpha \beta \mu_{1}} \lambda_{+}^{q} \mathcal{T}_{\beta}\right)+\rho^{(n)}\left(\overline{\mathcal{T}}_{\alpha} \gamma^{\alpha \beta \mu_{1}} \mathcal{T}_{\beta}\right) \operatorname{Tr}\left[\lambda_{+}^{q}\right]\right] p^{\mu_{2}} \cdots p^{\mu_{n}} \\
& +\left[\bar{\theta}^{(n)}\left(\overline{\mathcal{T}}_{\alpha} \gamma^{\mu_{1}} \gamma_{5} \lambda^{q} \mathcal{T}^{\alpha}\right)+\bar{\rho}^{(n)}\left(\overline{\mathcal{T}}_{\alpha} \gamma^{\mu_{1}} \gamma_{5} \mathcal{T}^{\alpha} \operatorname{Tr}[\lambda \underline{q}]\right] p^{\mu_{2}} \cdots p^{\mu_{n}}\right. \\
& -\sqrt{\frac{3}{2}} \bar{\omega}^{(n)}\left[\left(\overline{\mathcal{B}} \Theta^{\mu_{1} \mu} \lambda \underline{\mathcal{T}}_{\mu}\right)+\left(\overline{\mathcal{T}}_{\mu} \Theta^{\mu \mu_{1}} \lambda \underline{q} \mathcal{B}\right)\right] p^{\mu_{2}} \cdots p^{\mu_{n}}+\text { permutations }-\mathrm{Tr},
\end{aligned}
$$

where "Tr" denotes traces over Lorentz indices. In the first term of Eq. (11), the operator $U$ represents pseudoscalar meson fields $\phi$,

$$
U=\exp \left(i \frac{\sqrt{2} \phi}{f_{\phi}}\right)
$$

where $f_{\phi}$ is the pseudoscalar decay constant and the coefficients $a^{(n)}$ are related to moments of quark and antiquark PDFs in the pseudoscalar mesons. The flavor operators $\lambda_{ \pm}^{q}$ are defined by

$$
\lambda_{ \pm}^{q}=\frac{1}{2}\left(u \lambda^{q} u^{\dagger} \pm u^{\dagger} \lambda^{q} u\right),
$$

where $\lambda^{q}=\operatorname{diag}\left(\delta_{q u}, \delta_{q d}, \delta_{q s}\right)$ are diagonal $3 \times 3$ quark flavor matrices.

In the remaining terms of Eq. (11), the operators $\mathcal{B}$ and $\mathcal{T}_{\alpha}$ represent octet and decuplet baryon fields, respectively, and we define the Dirac tensors $\gamma^{\alpha \beta \rho}=\frac{1}{2}\left\{\gamma^{\mu \nu}, \gamma^{\alpha}\right\}$ and $\gamma^{\mu \nu}=\frac{1}{2}\left[\gamma^{\mu}, \gamma^{\nu}\right]$. The coefficients $\left\{\alpha^{(n)}, \beta^{(n)}, \sigma^{(n)}\right\}$ and $\left\{\bar{\alpha}^{(n)}, \bar{\beta}^{(n)}, \bar{\sigma}^{(n)}\right\}$ are related to moments of the spin-averaged and spin-dependent PDFs in octet baryons, while $\left\{\theta^{(n)}, \rho^{(n)}\right\}$ and $\left\{\bar{\theta}^{(n)}, \bar{\rho}^{(n)}\right\}$ are related to moments of spin-averaged and spin-dependent PDFs in decuplet baryons, respectively. The coefficients $\bar{\omega}^{(n)}$ are given in terms of moments of spindependent octet-decuplet transition PDFs, where the octetdecuplet transition tensor operator $\Theta^{\mu \nu}$ is defined as

$$
\Theta^{\mu \nu}=g^{\mu \nu}-\left(Z+\frac{1}{2}\right) \gamma^{\mu} \gamma^{\nu} .
$$

Here $Z$ is the decuplet off-shell parameter, and since physical quantities do not depend on $Z$, it is convenient to choose $Z=1 / 2$ to simplify the form of the spin- $3 / 2$ propagator $[28,29]$.

For the Kroll-Ruderman diagrams in Figs. 2(e), 2(f), 2(h), and 2(i), the presence of the pseudoscalar field at the vertex introduces hadronic axial vector operators, whose contribution to the quark axial vector operator can in general be written as

$$
\begin{aligned}
\mathcal{O}_{\Delta q}^{\mu_{1} \cdots \mu_{n}}= & {\left[\bar{\alpha}^{(n)}\left(\overline{\mathcal{B}} \gamma^{\mu_{1}} \gamma_{5} \mathcal{B} \lambda_{+}^{q}\right)+\bar{\beta}^{(n)}\left(\overline{\mathcal{B}}^{\mu_{1}} \gamma_{5} \lambda_{+}^{q} \mathcal{B}\right)+\bar{\sigma}^{(n)}\left(\overline{\mathcal{B}} \gamma^{\mu_{1}} \gamma_{5} \mathcal{B}\right) \operatorname{Tr}\left[\lambda_{+}^{q}\right]\right] p^{\mu_{2}} \cdots p^{\mu_{n}} } \\
& +\left[\alpha^{(n)}\left(\overline{\mathcal{B}}^{\mu_{1}} \mathcal{B} \lambda_{-}^{q}\right)+\beta^{(n)}\left(\overline{\mathcal{B}} \gamma^{\mu_{1}} \lambda_{-}^{q} \mathcal{B}\right)+\sigma^{(n)}\left(\overline{\mathcal{B}} \gamma^{\mu_{1}} \mathcal{B}\right) \operatorname{Tr}[\lambda \underline{q}]\right] p^{\mu_{2}} \cdots p^{\mu_{n}} \\
& +\left[\bar{\theta}^{(n)}\left(\overline{\mathcal{T}}_{\alpha} \gamma^{\mu_{1}} \gamma_{5} \lambda_{+}^{q} \mathcal{T}^{\alpha}\right)+\bar{\rho}^{(n)}\left(\overline{\mathcal{T}}_{\alpha} \gamma^{\mu_{1}} \gamma_{5} \mathcal{T}^{\alpha}\right) \operatorname{Tr}\left[\lambda_{+}^{q}\right]\right] p^{\mu_{2}} \cdots p^{\mu_{n}} \\
& +\left[\theta^{(n)}\left(\overline{\mathcal{T}}_{\alpha} \gamma^{\alpha \beta \mu_{1}} \lambda_{\underline{q}} \mathcal{T}_{\beta}\right)+\rho^{(n)}\left(\overline{\mathcal{T}}_{\alpha} \gamma^{\alpha \beta \mu_{1}} \mathcal{T}_{\beta}\right) \operatorname{Tr}\left[\lambda^{q}\right]\right] p^{\mu_{2}} \cdots p^{\mu_{n}} \\
& -\sqrt{\frac{3}{2}} \bar{\omega}^{(n)}\left[\left(\overline{\mathcal{B}} \Theta^{\mu_{1} \mu} \lambda_{+}^{q} \mathcal{T}_{\mu}\right)+\left(\overline{\mathcal{T}}_{\mu} \Theta^{\mu \mu_{1}} \lambda_{+}^{q} \mathcal{B}\right)\right] p^{\mu_{2}} \cdots p^{\mu_{n}}+\text { permutations }-\operatorname{Tr} .
\end{aligned}
$$

From the transformation properties of the operators $\mathcal{O}_{q}^{\mu_{1} \cdots \mu_{n}}$ and $\mathcal{O}_{\Delta q}^{\mu_{1} \cdots \mu_{n}}$ under parity [30], the sets of coefficients $\left\{\alpha^{(n)}, \beta^{(n)}, \sigma^{(n)}, \theta^{(n)}, \rho^{(n)}\right\}$ and $\left\{\bar{\alpha}^{(n)}, \bar{\beta}^{(n)}, \bar{\sigma}^{(n)}, \bar{\theta}^{(n)}, \bar{\rho}^{(n)}\right\}$ in (15) are the same as those in the spin-averaged operators in (11). 
The operators $\mathcal{B}$ and $\mathcal{T}_{\alpha}$ appearing in Eqs. (11) and (15) can be written in terms of the SU(3) baryon octet fields $B^{i j}$ (which include $N, \Lambda, \Sigma$, and $\Xi$ fields) and decuplet baryon fields $T_{\alpha}^{i j k}$ (which include $\Delta, \Sigma^{*}, \Xi^{*}$, and $\Omega$ fields) using the relations $[24,31]$

$$
(\overline{\mathcal{B}} \mathcal{B})=\operatorname{Tr}[\bar{B} B],
$$

$(\overline{\mathcal{B}} \mathcal{B} A)=-\frac{1}{6} \operatorname{Tr}[\bar{B} B A]+\frac{2}{3} \operatorname{Tr}[\bar{B} A B]+\frac{1}{6} \operatorname{Tr}[\bar{B} B] \operatorname{Tr}[A]$,

$(\overline{\mathcal{B}} A \mathcal{B})=-\frac{2}{3} \operatorname{Tr}[\bar{B} B A]-\frac{1}{3} \operatorname{Tr}[\bar{B} A B]+\frac{2}{3} \operatorname{Tr}[\bar{B} B] \operatorname{Tr}[A]$,

and

$$
\begin{aligned}
\left(\overline{\mathcal{T}}_{\alpha} \mathcal{T}_{\beta}\right) & =\bar{T}_{\alpha}^{k j i} T_{\beta}^{i j k}, \\
\left(\overline{\mathcal{T}}_{\alpha} A \mathcal{T}_{\beta}\right) & =\bar{T}_{\alpha}^{k j i} A^{i l} T_{\beta}^{l j k}, \\
\left(\overline{\mathcal{T}}_{\alpha} A \mathcal{B}\right) & =-\sqrt{\frac{2}{3}} \bar{T}_{\alpha}^{i j k} A^{i l} B^{j m} \varepsilon^{k l m},
\end{aligned}
$$

where $\varepsilon^{k l m}$ is the antisymmetric tensor. Applying the relations (16) and (17), the vector operator $\mathcal{O}_{q}^{\mu_{1} \cdots \mu_{n}}$ in Eq. (11) can then be more intuitively rearranged in the form

$$
\begin{aligned}
\mathcal{O}_{q}^{\mu_{1} \cdots \mu_{n}}= & Q_{\phi}^{(n-1)} \mathcal{O}_{\phi}^{\mu_{1} \cdots \mu_{n}}+Q_{B}^{(n-1)} \mathcal{O}_{B B^{\prime}}^{\mu_{1} \cdots \mu_{n}}+Q_{T}^{(n-1)} \mathcal{O}_{T T^{\prime}}^{\mu_{1} \cdots \mu_{n}} \\
& +Q_{(\operatorname{tad}) B \phi \phi}^{(n-1)} \mathcal{O}_{B \phi \phi}^{\mu_{1} \cdots \mu_{n}}+Q_{(\mathrm{KR}) B}^{(n-1)} \mathcal{O}_{B B^{\prime} \phi}^{\mu_{1} \cdots \mu_{n}} \\
& +Q_{(\mathrm{KR}) T}^{(n-1)} \mathcal{O}_{B T \phi}^{\mu_{1} \cdots \mu_{n}} .
\end{aligned}
$$

The individual vector hadronic operators in (18) are given by

$$
\begin{aligned}
\mathcal{O}_{\phi}^{\mu_{1} \cdots \mu_{n}} & =i^{n}\left(\bar{\phi} \partial^{\mu_{1}} \cdots \partial^{\mu_{n}} \phi-\phi \partial^{\mu_{1}} \cdots \partial^{\mu_{n}} \bar{\phi}\right), \\
\mathcal{O}_{B B^{\prime}}^{\mu_{1} \cdots \mu_{n}} & =\left(\bar{B}^{\prime} \gamma^{\mu_{1}} B\right) p^{\mu_{2}} \cdots p^{\mu_{n}}, \\
\mathcal{O}_{T T^{\prime}}^{\mu_{1} \cdots \mu_{n}} & =\left(\bar{T}_{\alpha}^{\prime} \gamma^{\alpha \beta \mu_{1}} T_{\beta}\right) p^{\mu_{2}} \cdots p^{\mu_{n}}, \\
\mathcal{O}_{B \phi \phi}^{\mu_{1} \cdots \mu_{n}} & =\frac{1}{f_{\phi}^{2}}\left(\bar{B} \gamma^{\mu_{1}} B \bar{\phi} \phi\right) p^{\mu_{2}} \cdots p^{\mu_{n}}, \\
\mathcal{O}_{B B^{\prime} \phi}^{\mu_{1} \cdots \mu_{n}} & =\frac{i}{f_{\phi}}\left(\bar{B}^{\prime} \gamma^{\mu_{1}} \gamma_{5} B \phi-\bar{B} \gamma^{\mu_{1}} \gamma_{5} B^{\prime} \bar{\phi}\right) p^{\mu_{2}} \cdots p^{\mu_{n}}, \\
\mathcal{O}_{B T \phi}^{\mu_{1} \cdots \mu_{n}} & =\frac{i}{f_{\phi}}\left(\bar{B} \Theta^{\mu_{1} \nu} T_{\nu} \bar{\phi}-\bar{T}_{\nu} \Theta^{\nu \mu_{1}} B \phi\right) p^{\mu_{2}} \cdots p^{\mu_{n}},
\end{aligned}
$$

and correspond to the insertions in the diagrams of Figs. 1, 2(d), 2(g), 2(j), 2(e), and 2(h), respectively. The coefficients $Q_{j}^{(n-1)}$ of each of the operators are defined in terms of
TABLE I. Moments $Q_{\phi}^{(n-1)}$ of the quark distributions $q(=u, d, s)$ in the pseudoscalar mesons $\pi^{+}, K^{+}$, and $K^{0}$. The moments are normalized such that $a^{(1)}=2$.

\begin{tabular}{lccc}
\hline \hline$\phi$ & $U_{\phi}^{(n-1)}$ & $D_{\phi}^{(n-1)}$ & $S_{\phi}^{(n-1)}$ \\
\hline$\pi^{+}$ & $\frac{1}{2} a^{(n)}$ & $-\frac{1}{2} a^{(n)}$ & 0 \\
$K^{+}$ & $\frac{1}{2} a^{(n)}$ & 0 & $-\frac{1}{2} a^{(n)}$ \\
$K^{0}$ & 0 & $\frac{1}{2} a^{(n)}$ & $-\frac{1}{2} a^{(n)}$ \\
\hline \hline
\end{tabular}

Mellin moments of the corresponding parton distributions in the intermediate mesons and baryons, as in Eq. (5),

$$
\begin{aligned}
Q_{\phi}^{(n-1)} & =\int_{-1}^{1} d x x^{n-1} q_{\phi}(x), \\
Q_{B}^{(n-1)} & =\int_{-1}^{1} d x x^{n-1} q_{B}(x), \\
Q_{T}^{(n-1)} & =\int_{-1}^{1} d x x^{n-1} q_{T}(x), \\
Q_{(\mathrm{tad}) B \phi \phi}^{(n-1)} & =\int_{-1}^{1} d x x^{n-1} q_{\phi}^{(\mathrm{tad}),} \\
Q_{(\mathrm{KR}) B}^{(n-1)} & =\int_{-1}^{1} d x x^{n-1} q_{B}^{(\mathrm{KR})}, \\
Q_{(\mathrm{KR}) T}^{(n-1)} & =\int_{-1}^{1} d x x^{n-1} q_{T}^{(\mathrm{KR}),}
\end{aligned}
$$

where the PDFs correspond to those appearing in the convolution expressions in Eqs. (9) and (10). Each of the moments $Q_{j}^{(n-1)}$ can be expressed in terms of the coefficients $\left\{a^{(n)} ; \alpha^{(n)}, \beta^{(n)}, \sigma^{(n)} ; \theta^{(n)}, \rho^{(n)}\right\}$ appearing in Eq. (11), as discussed below.

In particular, for the meson PDFs, the contributions to the $U_{\phi}^{(n-1)}, D_{\phi}^{(n-1)}$, and $S_{\phi}^{(n-1)}$ moments are listed in Table I for the $\phi=\pi^{+}, K^{+}$, and $K^{0}$ mesons. Conservation of the valence quark number fixes the normalization of the $n=1$ moment of the meson distribution, such that

$$
a^{(1)}=2 .
$$

Note that in the $\mathrm{SU}(3)$ symmetric limit, the $u$-quark moments in $\pi^{+}$and $K^{+}$are equivalent, as are the $s$-quark moments in $K^{+}$and $K^{0}$, while the $d$-quark moments in $\pi^{+}$ and $K^{0}$ have equal magnitude but opposite sign,

$$
\begin{aligned}
U_{\pi^{+}}^{(n-1)} & =-D_{\pi^{+}}^{(n-1)}=U_{K^{+}}^{(n-1)}=-S_{K^{+}}^{(n-1)}=D_{K^{0}}^{(n-1)} \\
& =-S_{K^{0}}^{(n-1)}=\frac{1}{2} a^{(n)} .
\end{aligned}
$$

The results for other charge states $\left(\pi^{-}, \pi^{0}, K^{-}\right.$, and $\left.\bar{K}^{0}\right)$ are obtained from those in Table I using charge symmetry. 
TABLE II. Moments $Q_{B}^{(n-1)}$ of the unpolarized quark distributions for $q=u, d$, or $s$ for octet baryons $B$. The spin-dependent moments $\Delta Q_{B}^{(n-1)}$ can be obtained from the entries here by the replacements $\left\{\alpha^{(n)} \rightarrow \bar{\alpha}^{(n)}, \beta^{(n)} \rightarrow \bar{\beta}^{(n)}, \sigma^{(n)} \rightarrow \bar{\sigma}^{(n)}\right\}$.

\begin{tabular}{|c|c|c|c|}
\hline$B$ & $U_{B}^{(n-1)}$ & $D_{B}^{(n-1)}$ & $S_{B}^{(n-1)}$ \\
\hline$p$ & $\frac{5}{6} \alpha^{(n)}+\frac{1}{3} \beta^{(n)}+\sigma^{(n)}$ & $\frac{1}{6} \alpha^{(n)}+\frac{2}{3} \beta^{(n)}+\sigma^{(n)}$ & $\sigma^{(n)}$ \\
\hline$n$ & $\frac{1}{6} \alpha^{(n)}+\frac{2}{3} \beta^{(n)}+\sigma^{(n)}$ & $\frac{5}{6} \alpha^{(n)}+\frac{1}{3} \beta^{(n)}+\sigma^{(n)}$ & $\sigma^{(n)}$ \\
\hline$\Sigma^{+}$ & $\frac{5}{6} \alpha^{(n)}+\frac{1}{3} \beta^{(n)}+\sigma^{(n)}$ & $\sigma^{(n)}$ & $\frac{1}{6} \alpha^{(n)}+\frac{2}{3} \beta^{(n)}+\sigma^{(n)}$ \\
\hline$\Sigma^{0}$ & $\frac{5}{12} \alpha^{(n)}+\frac{1}{6} \beta^{(n)}+\sigma^{(n)}$ & $\frac{5}{12} \alpha^{(n)}+\frac{1}{6} \beta^{(n)}+\sigma^{(n)}$ & $\frac{1}{6} \alpha^{(n)}+\frac{2}{3} \beta^{(n)}+\sigma^{(n)}$ \\
\hline$\Sigma^{-}$ & $\sigma^{(n)}$ & $\frac{5}{6} \alpha^{(n)}+\frac{1}{3} \beta^{(n)}+\sigma^{(n)}$ & $\frac{1}{6} \alpha^{(n)}+\frac{2}{3} \beta^{(n)}+\sigma^{(n)}$ \\
\hline$\Lambda$ & $\frac{1}{4} \alpha^{(n)}+\frac{1}{2} \beta^{(n)}+\sigma^{(n)}$ & $\frac{1}{4} \alpha^{(n)}+\frac{1}{2} \beta^{(n)}+\sigma^{(n)}$ & $\frac{1}{2} \alpha^{(n)}+\sigma^{(n)}$ \\
\hline$\Lambda \Sigma^{0}$ & $\frac{\sqrt{3}}{12}\left[\alpha^{(n)}-2 \beta^{(n)}\right]$ & $-\frac{\sqrt{3}}{12}\left[\alpha^{(n)}-2 \beta^{(n)}\right]$ & 0 \\
\hline$\Xi^{0}$ & $\frac{1}{6} \alpha^{(n)}+\frac{2}{3} \beta^{(n)}+\sigma^{(n)}$ & $\sigma^{(n)}$ & $\frac{5}{6} \alpha^{(n)}+\frac{1}{3} \beta^{(n)}+\sigma^{(n)}$ \\
\hline$\Xi^{-}$ & $\sigma^{(n)}$ & $\frac{1}{6} \alpha^{(n)}+\frac{2}{3} \beta^{(n)}+\sigma^{(n)}$ & $\frac{5}{6} \alpha^{(n)}+\frac{1}{3} \beta^{(n)}+\sigma^{(n)}$ \\
\hline
\end{tabular}

Unlike in baryons, the sea quark distributions in mesons are flavor symmetric. In the simplest valence quark models the sea quark distributions in pions and kaons are zero.

For the moments of the quark PDFs in the intermediate state baryons, the contributions from the $u, d$, and $s$ flavors to the octet baryon moments $Q_{B}^{(n-1)}$ are given in terms of combinations of $\left\{\alpha^{(n)}, \beta^{(n)}, \sigma^{(n)}\right\}$ and listed in Table II for baryons $B=p, n, \Sigma^{ \pm, 0}, \Lambda, \Xi^{-, 0}$ as well as for the $\Lambda-\Sigma^{0}$ interference. Solving for the coefficients, one can write these as linear combinations of the individual $u, d$, and $s$ quark moments in the proton,

$$
\begin{aligned}
& \alpha^{(n)}=\frac{4}{3} U_{p}^{(n-1)}-\frac{2}{3} D_{p}^{(n-1)}-\frac{2}{3} S_{p}^{(n-1)}, \\
& \beta^{(n)}=-\frac{1}{3} U_{p}^{(n-1)}+\frac{5}{3} D_{p}^{(n-1)}-\frac{4}{3} S_{p}^{(n-1)}, \\
& \sigma^{(n)}=S_{p}^{(n-1)} .
\end{aligned}
$$

Assuming the strangeness in the intermediate state nucleon to be zero (or equivalently, that the $u$ content of $\Sigma^{-}$, for example, vanishes), one finds for the lowest $(n=1)$ moments,

$$
\alpha^{(1)}=2, \quad \beta^{(1)}=1, \quad \sigma^{(1)}=0 .
$$

For the quark PDFs in the decuplet baryon intermediate states $T$, the moments $Q_{T}^{(n-1)}$ for the individual $u, d$, and $s$ flavors are given in terms of combinations of $\left\{\theta^{(n)}, \rho^{(n)}\right\}$, and are listed in Table III for $T=\Delta, \Sigma^{*}, \Xi^{*}$, and $\Omega^{-}$. Solving for the coefficients $\theta^{(n)}$ and $\rho^{(n)}$ in terms of the moments in the $\Delta^{+}$baryon, one has

$$
\begin{aligned}
& \theta^{(n)}=3\left(D_{\Delta^{+}}^{(n-1)}-S_{\Delta^{+}}^{(n-1)}\right)=\frac{3}{2}\left(U_{\Delta^{+}}^{(n-1)}-S_{\Delta^{+}}^{(n-1)}\right), \\
& \rho^{(n)}=S_{\Delta^{+}}^{(n-1)} .
\end{aligned}
$$

Again, assuming zero strangeness in the $\Delta^{+}$, the $n=1$ moments are given by

$$
\theta^{(1)}=3, \quad \rho^{(1)}=0 .
$$

For the moments of the distributions generated by the tadpole diagrams in Fig. 2(j), in Table IV we list the contributions $Q_{(\operatorname{tad}) B \phi \phi}^{(n-1)}$ for the $u, d$, and $s$ flavors in each octet baryon $B$. Note that the combinations involving $K^{0} \bar{K}^{0}$ do not contribute to the $u$-quark moments, those involving $K^{+} K^{-}$do not contribute to the $d$-quark moments, and the contributions from $\pi^{+} \pi^{-}$to the $s$-quark moments are also zero.

Finally, to complete the set of the contributions to the unpolarized PDFs, in Table $\mathrm{V}$ we list the moments $Q_{(\mathrm{KR}) B}^{(n-1)}$ and $Q_{(\mathrm{KR}) T}^{(n-1)}$ of the Kroll-Ruderman induced quark

TABLE III. Moments $Q_{T}^{(n-1)}$ of the unpolarized quark distributions for $q=u, d$, or $s$ for decuplet baryons $T$. The results for the spin-dependent moments $\Delta Q_{T}^{(n-1)}$ can be obtained by the replacements $\left\{\theta^{(n)} \rightarrow \bar{\theta}^{(n)}, \rho^{(n)} \rightarrow \bar{\rho}^{(n)}\right\}$.

\begin{tabular}{lccc}
\hline \hline$T$ & $U_{T}^{(n-1)}$ & $D_{T}^{(n-1)}$ & $S_{T}^{(n-1)}$ \\
\hline$\Delta^{++}$ & $\theta^{(n)}+\rho^{(n)}$ & $\rho^{(n)}$ & $\rho^{(n)}$ \\
$\Delta^{+}$ & $\frac{2}{3} \theta^{(n)}+\rho^{(n)}$ & $\frac{1}{3} \theta^{(n)}+\rho^{(n)}$ & $\rho^{(n)}$ \\
$\Delta^{0}$ & $\frac{1}{3} \theta^{(n)}+\rho^{(n)}$ & $\frac{2}{3} \theta^{(n)}+\rho^{(n)}$ & $\rho^{(n)}$ \\
$\Delta^{-}$ & $\rho^{(n)}$ & $\theta^{(n)}+\rho^{(n)}$ & $\rho^{(n)}$ \\
$\Sigma^{*+}$ & $\frac{2}{3} \theta^{(n)}+\rho^{(n)}$ & $\rho^{(n)}$ & $\frac{1}{3} \theta^{(n)}+\rho^{(n)}$ \\
$\Sigma^{* 0}$ & $\frac{1}{3} \theta^{(n)}+\rho^{(n)}$ & $\frac{1}{3} \theta^{(n)}+\rho^{(n)}$ & $\frac{1}{3} \theta^{(n)}+\rho^{(n)}$ \\
$\Sigma^{*-}$ & $\rho^{(n)}$ & $\frac{2}{3} \theta^{(n)}+\rho^{(n)}$ & $\frac{1}{3} \theta^{(n)}+\rho^{(n)}$ \\
$\Xi^{* 0}$ & $\frac{1}{3} \theta^{(n)}+\rho^{(n)}$ & $\rho^{(n)}$ & $\frac{2}{3} \theta^{(n)}+\rho^{(n)}$ \\
$\Xi^{*-}$ & $\rho^{(n)}$ & $\frac{1}{3} \theta^{(n)}+\rho^{(n)}$ & $\frac{2}{3} \theta^{(n)}+\rho^{(n)}$ \\
$\Omega^{-}$ & $\rho^{(n)}$ & $\rho^{(n)}$ & $\theta^{(n)}+\rho^{(n)}$ \\
\hline \hline
\end{tabular}


TABLE IV. Moments of the unpolarized $u, d$, and $s$ quark distributions in octet baryons $B$ arising from the $B B \phi \phi$ tadpole vertex, as in Fig. 2(j). The moments $U_{(\operatorname{tad}) B K^{0} \bar{K}^{0}}^{(n-1)}, D_{(\operatorname{tad}) B K^{+} K^{-}}^{(n-1)}$, and $S_{(\operatorname{tad}) B \pi^{+} \pi^{-}}^{(n-1)}$ are zero for all baryons $B$, and are not listed in the table.

\begin{tabular}{|c|c|c|c|c|c|c|}
\hline \multirow[b]{2}{*}{$B$} & \multicolumn{2}{|c|}{$U_{(\operatorname{tad}) B \phi \phi}^{(n-1)}$} & \multicolumn{2}{|c|}{$D_{(\operatorname{tad}) B \phi \phi}^{(n-1)}$} & \multicolumn{2}{|c|}{$S_{(\operatorname{tad}) B \phi \phi}^{(n-1)}$} \\
\hline & $\pi^{+} \pi^{-}$ & $K^{+} K^{-}$ & $\pi^{+} \pi^{-}$ & $K^{0} \overline{K^{0}}$ & $K^{0} \overline{K^{0}}$ & $K^{+} K^{-}$ \\
\hline$p$ & $-\frac{1}{3} \alpha^{(n)}+\frac{1}{6} \beta^{(n)}$ & $-\frac{5}{12} \alpha^{(n)}-\frac{1}{6} \beta^{(n)}$ & $\frac{1}{3} \alpha^{(n)}-\frac{1}{6} \beta^{(n)}$ & $-\frac{1}{12} \alpha^{(n)}-\frac{1}{3} \beta^{(n)}$ & $\frac{1}{12} \alpha^{(n)}+\frac{1}{3} \beta^{(n)}$ & $\frac{5}{12} \alpha^{(n)}+\frac{1}{6} \beta^{(n)}$ \\
\hline$n$ & $\frac{1}{3} \alpha^{(n)}-\frac{1}{6} \beta^{(n)}$ & $-\frac{1}{12} \alpha^{(n)}-\frac{1}{3} \beta^{(n)}$ & $-\frac{1}{3} \alpha^{(n)}+\frac{1}{6} \beta^{(n)}$ & $-\frac{5}{12} \alpha^{(n)}-\frac{1}{6} \beta^{(n)}$ & $\frac{5}{12} \alpha^{(n)}+\frac{1}{6} \beta^{(n)}$ & $\frac{1}{12} \alpha^{(n)}+\frac{1}{3} \beta^{(n)}$ \\
\hline$\Sigma^{+}$ & $-\frac{5}{12} \alpha^{(n)}-\frac{1}{6} \beta^{(n)}$ & $-\frac{1}{3} \alpha^{(n)}+\frac{1}{6} \beta^{(n)}$ & $\frac{5}{12} \alpha^{(n)}+\frac{1}{6} \beta^{(n)}$ & $\frac{1}{12} \alpha^{(n)}+\frac{1}{3} \beta^{(n)}$ & $-\frac{1}{12} \alpha^{(n)}-\frac{1}{3} \beta^{(n)}$ & $\frac{1}{3} \alpha^{(n)}-\frac{1}{6} \beta^{(n)}$ \\
\hline$\Sigma^{0}$ & 0 & $-\frac{1}{8} \alpha^{(n)}+\frac{1}{4} \beta^{(n)}$ & 0 & $-\frac{1}{8} \alpha^{(n)}+\frac{1}{4} \beta^{(n)}$ & $\frac{1}{8} \alpha^{(n)}-\frac{1}{4} \beta^{(n)}$ & $\frac{1}{8} \alpha^{(n)}-\frac{1}{4} \beta^{(n)}$ \\
\hline$\Sigma^{-}$ & $\frac{5}{12} \alpha^{(n)}+\frac{1}{6} \beta^{(n)}$ & $\frac{1}{12} \alpha^{(n)}+\frac{1}{3} \beta^{(n)}$ & $-\frac{5}{12} \alpha^{(n)}-\frac{1}{6} \beta^{(n)}$ & $-\frac{1}{3} \alpha^{(n)}+\frac{1}{6} \beta^{(n)}$ & $\frac{1}{3} \alpha^{(n)}-\frac{1}{6} \beta^{(n)}$ & $-\frac{1}{12} \alpha^{(n)}-\frac{1}{3} \beta^{(n)}$ \\
\hline$\Lambda$ & 0 & $\frac{1}{8} \alpha^{(n)}-\frac{1}{4} \beta^{(n)}$ & 0 & $\frac{1}{8} \alpha^{(n)}-\frac{1}{4} \beta^{(n)}$ & $-\frac{1}{8} \alpha^{(n)}+\frac{1}{4} \beta^{(n)}$ & $-\frac{1}{8} \alpha^{(n)}+\frac{1}{4} \beta^{(n)}$ \\
\hline$\Xi^{0}$ & $-\frac{1}{12} \alpha^{(n)}-\frac{1}{3} \beta^{(n)}$ & $\frac{1}{3} \alpha^{(n)}-\frac{1}{6} \beta^{(n)}$ & $\frac{1}{12} \alpha^{(n)}+\frac{1}{3} \beta^{(n)}$ & $\frac{5}{12} \alpha^{(n)}+\frac{1}{6} \beta^{(n)}$ & $-\frac{5}{12} \alpha^{(n)}-\frac{1}{6} \beta^{(n)}$ & $-\frac{1}{3} \alpha^{(n)}+\frac{1}{6} \beta^{(n)}$ \\
\hline$\Xi^{-}$ & $\frac{1}{12} \alpha^{(n)}+\frac{1}{3} \beta^{(n)}$ & $\frac{5}{12} \alpha^{(n)}+\frac{1}{6} \beta^{(n)}$ & $-\frac{1}{12} \alpha^{(n)}-\frac{1}{3} \beta^{(n)}$ & $\frac{1}{3} \alpha^{(n)}-\frac{1}{6} \beta^{(n)}$ & $-\frac{1}{3} \alpha^{(n)}+\frac{1}{6} \beta^{(n)}$ & $-\frac{5}{12} \alpha^{(n)}-\frac{1}{6} \beta^{(n)}$ \\
\hline
\end{tabular}

distributions from Figs. 2(e) and 2(h), for the transitions from a proton initial state to intermediate states including octet $B$ and decuplet $T$ baryons, respectively. (Similar results can be derived for other octet or decuplet baryon initial states, but are not listed here to avoid unnecessary detail.) Note that, unlike for all other contributions from the diagrams in Fig. 2, the moments $Q_{(\mathrm{KR}) B}^{(n-1)}$ and $Q_{(\mathrm{KR}) T}^{(n-1)}$ are given in terms of the coefficients $\bar{\alpha}^{(n)}, \bar{\beta}^{(n)}$, and $\bar{\omega}^{(n)}$, which are related to moments of the spin-dependent parton distributions.

For the latter, recall that spin-dependent PDFs are related to matrix elements of the axial vector operators $\mathcal{O}_{\Delta q}^{\mu_{1} \cdots \mu_{n}}$ in Eq. (15), which, using the relations (16) and (17), can be expanded in terms of hadronic axial vector operators with

TABLE V. Moments of the unpolarized $u, d$, and $s$ quark distributions from the Kroll-Ruderman vertex for transitions from a proton initial state to octet and decuplet baryon intermediate states, as in Figs. 2(e) and 2(h), respectively.

\begin{tabular}{lccc}
\hline \hline$B \phi$ & $U_{(\mathrm{KR}) B}^{(n-1)}$ & $D_{(\mathrm{KR}) B}^{(n-1)}$ & $S_{(\mathrm{KR}) B}^{(n-1)}$ \\
\hline$n \pi^{+}$ & $-\frac{\sqrt{2}}{3} \bar{\alpha}^{(n)}+\frac{\sqrt{2}}{6} \bar{\beta}^{(n)}$ & $\frac{\sqrt{2}}{3} \bar{\alpha}^{(n)}-\frac{\sqrt{2}}{6} \bar{\beta}^{(n)}$ & 0 \\
$\Sigma^{0} K^{+}$ & $\frac{1}{12} \bar{\alpha}^{(n)}+\frac{1}{3} \bar{\beta}^{(n)}$ & 0 & $-\frac{1}{12} \bar{\alpha}^{(n)}-\frac{1}{3} \bar{\beta}^{(n)}$ \\
$\Sigma^{+} K^{0}$ & 0 & $\frac{\sqrt{2}}{12} \bar{\alpha}^{(n)}+\frac{\sqrt{2}}{3} \bar{\beta}^{(n)}$ & $-\frac{1}{12} \bar{\alpha}^{(n)}-\frac{1}{3} \bar{\beta}^{(n)}$ \\
$\Lambda^{0} K^{+}$ & $\frac{\sqrt{3}}{4} \bar{\alpha}^{(n)}$ & 0 & $-\frac{\sqrt{3}}{4} \bar{\alpha}^{(n)}$ \\
\hline$T \phi$ & $U_{(\mathrm{KR}) T}^{(n-1)}$ & $D_{(\mathrm{KR}) T}^{(n-1)}$ & $S_{(\mathrm{KR}) T}^{(n-1)}$ \\
\hline$\Delta^{0} \pi^{+}$ & $\frac{1}{\sqrt{6}} \bar{\omega}^{(n)}$ & $-\frac{1}{\sqrt{6}} \bar{\omega}^{(n)}$ & 0 \\
$\Delta^{++} \pi^{-}$ & $\frac{1}{\sqrt{2}} \bar{\omega}^{(n)}$ & $-\frac{1}{\sqrt{2}} \bar{\omega}^{(n)}$ & 0 \\
$\Sigma^{* 0} K^{+}$ & $\frac{1}{2 \sqrt{3}} \bar{\omega}^{(n)}$ & 0 & $-\frac{1}{2 \sqrt{3}} \bar{\omega}^{(n)}$ \\
$\Sigma^{*+} K^{0}$ & 0 & $-\frac{1}{\sqrt{6}} \bar{\omega}^{(n)}$ & $\frac{1}{\sqrt{6}} \bar{\omega}^{(n)}$ \\
\hline \hline
\end{tabular}

coefficients given by moments $\Delta Q_{j}^{(n-1)}$ of the spin-dependent distributions. In analogy to the expansion in Eq. (18), we therefore expand the axial vector operators as

$$
\begin{aligned}
\mathcal{O}_{\Delta q}^{\mu_{1} \cdots \mu_{n}}= & \Delta Q_{B}^{(n-1)} \tilde{\mathcal{O}}_{B B^{\prime}}^{\mu_{1} \cdots \mu_{n}}+\Delta Q_{T}^{(n-1)} \tilde{\mathcal{O}}_{T T^{\prime}}^{\mu_{1} \cdots \mu_{n}} \\
& +\Delta Q_{B T}^{(n-1)} \tilde{\mathcal{O}}_{B T}^{\mu_{1} \cdots \mu_{n}}+\cdots,
\end{aligned}
$$

where only the operators relevant for the calculation of unpolarized PDFs are listed. [The remaining terms not listed in Eq. (27) will be relevant for the calculation of spindependent PDFs in the proton [32].] More explicitly, the axial vector hadronic operators in (27) are given by

$$
\begin{aligned}
& \tilde{\mathcal{O}}_{B B^{\prime}}^{\mu_{1} \cdots \mu_{n}}=\left(\bar{B}^{\prime} \gamma^{\mu_{1}} \gamma_{5} B\right) p^{\mu_{2}} \cdots p^{\mu_{n}}, \\
& \tilde{\mathcal{O}}_{T T^{\prime}}^{\mu_{1} \cdots \mu_{n}}=\left(\bar{T}^{\prime}{ }_{\alpha} \gamma^{\mu_{1}} \gamma_{5} T^{\alpha}\right) p^{\mu_{2}} \cdots p^{\mu_{n}}, \\
& \tilde{\mathcal{O}}_{B T}^{\mu_{1} \cdots \mu_{n}}=\left(\bar{B} \Theta^{\mu_{1} \nu} T_{\nu}+\bar{T}_{\nu} \Theta^{\nu \mu_{1}} B\right) p^{\mu_{2}} \cdots p^{\mu_{n}},
\end{aligned}
$$

with the corresponding moments $\Delta Q_{j}^{(n-1)}$ of the spindependent PDFs defined by

$$
\begin{aligned}
& \Delta Q_{B}^{(n-1)}=\int_{-1}^{1} d x x^{n-1} \Delta q_{B}(x), \\
& \Delta Q_{T}^{(n-1)}=\int_{-1}^{1} d x x^{n-1} \Delta q_{T}(x), \\
& \Delta Q_{B T}^{(n-1)}=\int_{-1}^{1} d x x^{n-1} \Delta q_{B T}(x) .
\end{aligned}
$$

For simplicity, in Eq. (29) we restrict ourselves to the diagonal octet $\left(B=B^{\prime}\right)$ and diagonal decuplet $\left(T=T^{\prime}\right)$ cases, with respective spin-dependent PDFs $\Delta q_{B}(x)$ and 
TABLE VI. Moments $\Delta Q_{B T}^{(n-1)}$ of the polarized $u, d$, and $s$ quark distributions from the axial octet-decuplet transition.

\begin{tabular}{lccc}
\hline \hline$B T$ & $\Delta U_{B T}^{(n-1)}$ & $\Delta D_{B T}^{(n-1)}$ & $\Delta S_{B T}^{(n-1)}$ \\
\hline$n \Delta^{0}$ & $-\frac{1}{\sqrt{3}} \bar{\omega}^{(n)}$ & $\frac{1}{\sqrt{3}} \bar{\omega}^{(n)}$ & 0 \\
$p \Delta^{+}$ & $-\frac{1}{\sqrt{3}} \bar{\omega}^{(n)}$ & $\frac{1}{\sqrt{3}} \bar{\omega}^{(n)}$ & 0 \\
$\Sigma^{0} \Sigma^{* 0}$ & $-\frac{1}{2 \sqrt{3}} \bar{\omega}^{(n)}$ & $-\frac{1}{2 \sqrt{3}} \bar{\omega}^{(n)}$ & $\frac{1}{\sqrt{3}} \bar{\omega}^{(n)}$ \\
$\Sigma^{+} \Sigma^{*+}$ & $\frac{1}{\sqrt{3}} \bar{\omega}^{(n)}$ & 0 & $-\frac{1}{\sqrt{3}} \bar{\omega}^{(n)}$ \\
$\Sigma^{-} \Sigma^{*-}$ & 0 & $-\frac{1}{\sqrt{3}} \bar{\omega}^{(n)}$ & $\frac{1}{\sqrt{3}} \bar{\omega}^{(n)}$ \\
$\Lambda \Sigma^{* 0}$ & $\frac{1}{2} \bar{\omega}^{(n)}$ & $-\frac{1}{2} \bar{\omega}^{(n)}$ & 0 \\
\hline \hline
\end{tabular}

$\Delta q_{T}(x)$, and the octet-decuplet transition distribution, $\Delta q_{B T}(x)$. In particular, the moments $\Delta Q_{B}^{(n-1)}$ of the spindependent PDFs in octet baryons can be obtained from the entries in Table II by substituting $\left\{\alpha^{(n)} \rightarrow \bar{\alpha}^{(n)}, \beta^{(n)} \rightarrow \bar{\beta}^{(n)}\right.$, $\left.\sigma^{(n)} \rightarrow \bar{\sigma}^{(n)}\right\}$, while the moments $\Delta Q_{T}^{(n-1)}$ of the spindependent PDFs in decuplet baryons are obtained from Table III with the replacements $\left\{\theta^{(n)} \rightarrow \bar{\theta}^{(n)}, \rho^{(n)} \rightarrow \bar{\rho}^{(n)}\right\}$. For the octet-decuplet axial transition distribution, the moments, $\Delta Q_{B T}^{(n-1)}$, are given in terms of the coefficient $\bar{\omega}$ in Eq. (15) and are listed in Table VI. Solving for the octet coefficients in terms of the moments of the spin-dependent proton PDFs in the proton, one has, in analogy with Eq. (23), the relations

$$
\begin{aligned}
& \bar{\alpha}^{(n)}=\frac{4}{3} \Delta U_{p}^{(n-1)}-\frac{2}{3} \Delta D_{p}^{(n-1)}-\frac{2}{3} \Delta S_{p}^{(n-1)}, \\
& \bar{\beta}^{(n)}=-\frac{1}{3} \Delta U_{p}^{(n-1)}+\frac{5}{3} \Delta D_{p}^{(n-1)}-\frac{4}{3} \Delta S_{p}^{(n-1)}, \\
& \bar{\sigma}^{(n)}=\Delta S_{p}^{(n-1)} .
\end{aligned}
$$

Similarly, for the decuplet case, the coefficients $\bar{\theta}^{(n)}$ and $\bar{\rho}^{(n)}$ can be written in terms of the moments of the spindependent PDFs of quarks in the $\Delta^{+}$bayron,

$\bar{\theta}^{(n)}=3\left(\Delta D_{\Delta^{+}}^{(n-1)}-\Delta S_{\Delta^{+}}^{(n-1)}\right)=\frac{3}{2}\left(\Delta U_{\Delta^{+}}^{(n-1)}-\Delta S_{\Delta^{+}}^{(n-1)}\right)$,

$\bar{\rho}^{(n)}=\Delta S_{\Delta^{+}}^{(n-1)}$.

The moments of the octet-decuplet transition operators can be related to the moments of the octet baryon operators via the $\mathrm{SU}(3)$ relation

$$
\bar{\omega}^{(n)}=-\frac{1}{2} \bar{\alpha}^{(n)}+\bar{\beta}^{(n)},
$$

for all $n$. For the $n=1$ octet baryon moments, in particular, the coefficients are given in terms of axial vector charges $F$ and $D$, $\bar{\alpha}^{(1)}=\frac{2}{3}(3 F+D), \quad \bar{\beta}^{(1)}=\frac{1}{3}(3 F-5 D), \quad \bar{\sigma}^{(1)}=0$.

In terms of moments of the spin-dependent proton PDFs, for the octet-decuplet transition vertex, $\bar{\omega}^{(1)}$ is given by the SU(3) symmetry relation [27],

$$
\bar{\omega}^{(1)}=-\Delta U_{p}^{(0)}+2 \Delta D_{p}^{(0)}-\Delta S_{p}^{(0)},
$$

which also reproduces the relation $\mathcal{C}=-2 D$ between the meson-octet-decuplet baryon coupling $\mathcal{C}$ and the mesonoctet coupling $D$ [33]. Note that through Eq. (32) the quark distributions in the Kroll-Ruderman diagrams with decuplet baryon intermediate states in Fig. 2(h) are related to the spin-dependent distributions of quarks in proton.

This completes the discussion of the moments of the PDFs of the various mesons and baryons appearing in the intermediate states in the diagrams of Fig. 2. From these, in the next section we derive relations for the $x$ dependence of the PDFs themselves.

\section{B. SU(3) relations for baryon and meson PDFs}

In the previous section we derived relations between the coefficients of the various operators in $\mathcal{O}_{q}^{\mu_{1} \cdots \mu_{n}}$ and $\mathcal{O}_{\Delta q}^{\mu_{1} \cdots \mu_{n}}$ and the $n$th Mellin moments of the quark distributions in Eqs. (22)-(26) and Eqs. (30)-(34). Since these relations are valid for all moments $n$, one can derive from them explicit expressions for the $x$ dependence of the PDFs.

For the valence distributions in the pion and kaon, from Eq. (22) and Table I one has

$$
\begin{aligned}
\bar{q}_{\pi}(x) & \equiv u_{\pi^{+}}(x)=\bar{d}_{\pi^{+}}(x)=d_{\pi^{-}}(x)=\bar{u}_{\pi^{-}}(x) \\
& =u_{K^{+}}(x)=\bar{s}_{K^{+}}(x)=d_{K^{0}}(x)=\bar{s}_{K^{0}}(x),
\end{aligned}
$$

for all values of $x$. For the PDFs in the baryons, to simplify notations we shall label the bare distributions in the proton without an explicit baryon subscript, $q(x) \equiv q_{p}(x)$, and those in the $\Delta^{+}$baryon by $q_{\Delta}(x) \equiv q_{\Delta^{+}}(x)$. Starting with the quark distributions in the $\mathrm{SU}(3)$ octet baryons, from Table II the individual $u$-, $d$-, and $s$-quark flavor PDFs can be written in terms of the proton PDFs as

$$
\begin{aligned}
u_{n}(x) & =d(x), \quad d_{n}(x)=u(x), \quad s_{n}(x)=s(x), \\
u_{\Sigma^{+}}(x) & =u(x), \quad d_{\Sigma^{+}}(x)=s(x), \quad s_{\Sigma^{+}}(x)=d(x), \\
u_{\Sigma^{0}}(x) & =\frac{1}{2}[u(x)+s(x)], \quad s_{\Sigma^{0}}(x)=d(x), \\
d_{\Sigma^{0}}(x) & =u_{\Sigma^{0}}(x), \quad d_{\Sigma^{-}}(x)=d(x), \\
u_{\Sigma^{-}}(x) & =s(x), \quad d_{\Sigma^{-}}(x)=u(x), \quad \\
u_{\Lambda}(x) & =\frac{1}{6}[4 d(x)+u(x)+s(x)], \\
s_{\Lambda}(x) & =\frac{1}{3}[2 u(x)-d(x)+2 s(x)]
\end{aligned}
$$


For the quark distributions in the $\mathrm{SU}(3)$ decuplet baryons, from Table III the $u-, d$-, and $s$-quark PDFs can be written in terms of the PDFs in the $\Delta^{+}$as

$$
\begin{aligned}
& u_{\Delta^{++}}(x)=u_{\Delta}(x)+d_{\Delta}(x)-s_{\Delta}(x), \\
& d_{\Delta^{++}}(x)=s_{\Delta}(x), \quad s_{\Delta^{++}}(x)=s_{\Delta}(x), \\
& u_{\Delta^{0}}(x)=d_{\Delta}(x), \quad d_{\Delta^{0}}(x)=u_{\Delta}(x), \quad s_{\Delta^{0}}(x)=s_{\Delta}(x), \\
& u_{\Delta^{-}}(x)=s_{\Delta}(x), \quad d_{\Delta^{-}}(x)=u_{\Delta^{++}}(x), \quad s_{\Delta^{-}}(x)=s_{\Delta}(x), \\
& u_{\Sigma^{*+}}(x)=u_{\Delta}(x), \quad d_{\Sigma^{*+}}(x)=s_{\Delta}(x), \quad s_{\Sigma^{*+}}(x)=d_{\Delta}(x), \\
& u_{\Sigma^{* 0}}(x)=d_{\Delta}(x), \quad d_{\Sigma^{* 0}}(x)=d_{\Delta}(x), \quad s_{\Sigma^{* 0}}(x)=d_{\Delta}(x), \\
& u_{\Sigma^{*-}}(x)=s_{\Delta}(x), \\
& s_{\Sigma^{*-}}(x)=d_{\Delta}(x) .
\end{aligned}
$$

In our actual numerical calculations for the strange asymmetry, for simplicity we approximate $d_{\Delta}(x) \approx d(x)$ and assume valence quark dominance for the bare states, so that $s(x) \approx s_{\Delta}(x) \approx 0$. Numerically we find that the octet baryon contributions to the $s-\bar{s}$ asymmetry are in fact dominant, and the approximations for the input PDFs of the decuplet states do not affect the conclusions of our analysis.

For the PDFs arising from the tadpole diagrams in Fig. 2(j), from Table IV the $u$-, $d$-, and $s$-quark distributions can be written as

$$
\begin{aligned}
& u_{\pi^{+}}^{(\mathrm{tad})}(x)=d_{\pi^{+}}^{(\mathrm{tad})}(x)=u(x)-d(x), \quad s_{\pi^{+}}^{(\mathrm{tad})}(x)=0, \\
& u_{K^{+}}^{(\mathrm{tad})}(x)=s_{K^{+}}^{(\mathrm{tad})}(x)=\frac{1}{2}[u(x)-s(x)], \quad d_{K^{+}}^{(\mathrm{tad})}(x)=0, \\
& d_{K^{0}}^{(\mathrm{tad})}(x)=s_{K^{0}}^{(\mathrm{tad})}(x)=d(x)-s(x), \quad u_{K^{0}}^{(\mathrm{tad})}(x)=0 .
\end{aligned}
$$

The distributions associated with the tadpole gauge link diagrams in Fig. 2(g) turn out to be the same as those for the regular tadpole diagrams,

$$
q_{\phi}^{(\delta)}(x)=q_{\phi}^{(\operatorname{tad})}(x) .
$$

Turning now to the Kroll-Ruderman diagrams in Figs. 2(e) and 2(h), for a proton initial state the corresponding PDFs are expressed in terms of spin-dependent
PDFs in the proton, $\Delta q(x) \equiv \Delta q_{p}(x)$. From Table V, for the octet baryon intermediate states the $u$-, $d$-, and $s$-quark distributions are given by

$$
\begin{aligned}
& u_{n}^{(\mathrm{KR})}(x)=d_{n}^{(\mathrm{KR})}(x)=\frac{\Delta u(x)-\Delta d(x)}{F+D}, \quad s_{n}^{(\mathrm{KR})}(x)=0, \\
& d_{\Sigma^{+}}^{(\mathrm{KR})}(x)=s_{\Sigma^{+}}^{(\mathrm{KR})}(x)=\frac{\Delta d(x)-\Delta s(x)}{F-D}, \quad u_{\Sigma^{+}}^{(\mathrm{KR})}(x)=0,
\end{aligned}
$$

$$
u_{\Sigma^{0}}^{(\mathrm{KR})}(x)=s_{\Sigma^{0}}^{(\mathrm{KR})}(x)=\frac{\Delta d(x)-\Delta s(x)}{F-D}, \quad d_{\Sigma^{0}}^{(\mathrm{KR})}(x)=0,
$$

$$
\begin{aligned}
& u_{\Lambda}^{(\mathrm{KR})}(x)=s_{\Lambda}^{(\mathrm{KR})}(x)=\frac{2 \Delta u(x)-\Delta d(x)-\Delta s(x)}{3 F+D}, \\
& d_{\Lambda}^{(\mathrm{KR})}(x)=0 .
\end{aligned}
$$

Similarly, for the decuplet baryon intermediate states the individual quark flavor Kroll-Ruderman distributions are given by

$u_{\Delta^{++}}^{(\mathrm{KR})}(x)=d_{\Delta^{++}}^{(\mathrm{KR})}(x)=\frac{\Delta u(x)-2 \Delta d(x)+\Delta s(x)}{2 D}$,
$s_{\Delta^{++}}^{(\mathrm{KR})}(x)=0$,

$u_{\Delta^{0}}^{(\mathrm{KR})}(x)=d_{\Delta^{0}}^{(\mathrm{KR})}(x)=\frac{\Delta u(x)-2 \Delta d(x)+\Delta s(x)}{2 D}$,

$s_{\Delta^{0}}^{(\mathrm{KR})}(x)=0$,

$d_{\Sigma^{*+}}^{(\mathrm{KR})}(x)=s_{\Sigma^{*+}}^{(\mathrm{KR})}(x)=\frac{\Delta u(x)-2 \Delta d(x)+\Delta s(x)}{2 D}$,

$u_{\Sigma^{*+}}^{(\mathrm{KR})}(x)=0$,

$u_{\Sigma^{* 0}}^{(\mathrm{KR})}(x)=s_{\Sigma^{* 0}}^{(\mathrm{KR})}(x)=\frac{\Delta u(x)-2 \Delta d(x)+\Delta s(x)}{2 D}$,

$d_{\Sigma^{* 0}}^{(\mathrm{KR})}(x)=0$.

The PDFs associated with the KR gauge link diagrams in Figs. 2(f) and 2(i) are the same as those for the regular KR diagrams,

$$
\begin{aligned}
& q_{B}^{(\delta)}(x)=q_{B}^{(\mathrm{KR})}(x), \\
& q_{T}^{(\delta)}(x)=q_{T}^{(\mathrm{KR})}(x) .
\end{aligned}
$$


With this set of distributions in the SU(3) octet and decuplet baryons and mesons, and the proton $\rightarrow$ meson + baryon splitting functions from Ref. [20], we can finally proceed with the computation of the meson loop contributions to the quark and antiquark PDFs in the proton, as in Eqs. (9) and (10). In the following section we focus on the calculation of specific PDF asymmetries in the proton numerically.

\section{SEA QUARK ASYMMETRIES IN THE PROTON}

To illustrate the calculation of the contributions to PDFs from pseudoscalar meson loops within the nonlocal chiral effective theory framework, we consider the examples of the flavor asymmetry in the light antiquark sea in the proton, $\bar{d}-\bar{u}$, and the strange-antistrange asymmetry in the nucleon, $s-\bar{s}$. In both quantities perturbatively generated contributions from gluon radiation effectively cancel, at least up to next-to-next-to-leading order corrections in $\alpha_{s}$ [13], so that observation of large asymmetries may be indicative of nonperturbative effects [14-16].

For the numerical calculation of the meson-bayron splitting functions, earlier work used various regularization prescriptions, including sharp transverse momentum cutoffs, Pauli-Villars regularization, as well as phenomenological vertex form factors [24,25,34-37]. At times the prescriptions have been imposed in rather ad hoc ways, without necessarily ensuring that the relevant symmetries, such as Lorentz, chiral, and local gauge symmetries, are necessarily respected. In the present work we for the first time perform the calculation within nonlocal regularization, which is consistent with all of the above symmetry requirements. An advantage of the nonlocal method is that only a single parameter, $\Lambda$, is needed to regulate all of the onshell, off-shell, and $\delta$ functions associated with each of the diagrams in Figs. 1 and 2.

Following Ref. [20], in the present analysis we adopt a dipole shape in the meson virtuality $k^{2}$ for the regulator functions for the one-loop contributions, parametrized by a cutoff parameter $\Lambda$,

$$
\widetilde{F}(k)=\left(\frac{\Lambda^{2}-m_{\phi}^{2}}{D_{\Lambda}}\right)^{2},
$$

where $D_{\Lambda}=k^{2}-\Lambda^{2}+i \varepsilon$. The cutoff $\Lambda$ can be determined by fitting the calculated meson-exchange cross section to differential cross sections data for inclusive baryon production in high-energy $p p$ scattering, $p p \rightarrow B X$, for different species of baryon $B$. Summing over the particles $X$ in the final state, the differential inclusive baryon production cross sections can be written as

$$
\sigma\left(y, k_{\perp}^{2}\right) \equiv E \frac{d^{3} \sigma}{d^{3} k}=\frac{\bar{y}}{\pi} \frac{d^{2} \sigma}{d y d k_{\perp}^{2}},
$$

where $E$ is the incident proton energy and $\bar{y} \equiv 1-y$ is the longitudinal momentum fraction of the incident proton carried by the produced baryon $B$. In Eq. (44) we have used the fact that for spin-averaged scattering the differential cross section is independent of the azimuthal angle. Available data exist on inclusive neutron and $\Delta^{++}$production [38-40], as well as on $\Lambda$ and $\Sigma^{*+}$ production [39,41,42] in the hyperon sector. In principle, the cutoffs may depend on the baryon $B$, although within the $\mathrm{SU}(3)$ symmetry framework we do not expect large variations among the different $\Lambda$ values.

Once the cutoffs are determined and the one-loop splitting functions are fixed, these can then be convoluted with the various meson and baryon PDFs in Eqs. (9) and (10) to compute the contributions to the PDFs in the proton. In the numerical calculations the input PDFs of the pion and kaon are taken from Aicher et al. [43]. The spinaveraged PDFs of the proton are from Ref. [44], while the spin-dependent PDFs are taken from Ref. [45]. Since the valence pion and proton PDFs are reasonably well determined, at least compared with the sea quark distributions, using other pion [46-50] or proton [51,52] parametrizations will not lead to significant differences.

\section{A. $\bar{d}-\bar{u}$ asymmetry}

Turning to the light antiquark asymmetry in the proton sea, within the chiral effective theory framework the primary source of the asymmetry is the meson rainbow and bubble diagrams in Fig. 1. In this approximation the $\bar{d}-\bar{u}$ difference does not depend directly on the structure of the baryon coupling diagrams in Fig. 2, but only on the splitting functions and the substructure of the pion. More specifically, from Eq. (9) one can write the contribution to the $\bar{d}-\bar{u}$ difference in the proton as the convolution

$\bar{d}(x)-\bar{u}(x)=\left[\left(f_{\pi^{+} n}^{(\mathrm{rbw})}+f_{\pi^{+} \Delta^{0}}^{(\mathrm{rbw})}-f_{\pi^{-} \Delta^{++}}^{(\mathrm{rbw})}+f_{\pi}^{(\mathrm{bub})}\right) \otimes \bar{q}_{\pi}\right](x)$,

where the first (octet rainbow) term in the brackets is from Fig. 1(a), the second and third (decuplet rainbow) terms correspond to Fig. 1(b), and the fourth (bubble) term is from Fig. 1(c). Using the notations of Ref. [20], the splitting functions in Eq. (45) for the rainbow and bubble diagrams can be expressed in terms of octet and decuplet basis functions. In particular, for the $\pi N$ configuration the function $f_{\pi^{+} n}^{(\mathrm{rbw})}$ is given by a sum of nucleon on-shell and $\delta$ function contributions,

$$
f_{\pi^{+} n}^{(\mathrm{rbw})}(y)=\frac{2(D+F)^{2} M^{2}}{(4 \pi f)^{2}}\left[f_{N}^{(\mathrm{on})}(y)+f_{\pi}^{(\delta)}(y)-\delta f_{\pi}^{(\delta)}(y)\right],
$$


where $D$ and $F$ are the $\mathrm{SU}(3)$ flavor coefficients, and $f=$ $93 \mathrm{MeV}$ is the pseudoscalar meson decay constant. Explicit forms for the basis functions are given in Ref. [20] for the dipole regulator $\tilde{F}(k)$ in Eq. (43). The on-shell function $f_{N}^{(\text {on })}$ is nonzero for $y>0$, while the local $f_{\pi}^{(\delta)}$ and nonlocal $\delta f_{\pi}^{(\delta)}$ functions are proportional to $\delta(y)$ [20], and therefore contribute to the $\bar{d}-\bar{u}$ asymmetry only at $x=0[34,35]$. In the pointlike limit, in which the form factor cutoff $\Lambda \rightarrow \infty$, the nonlocal function $\delta f_{\pi}^{(\delta)}$ vanishes; however, at finite $\Lambda$ values it remains nonzero.

In the standard formulation of the chiral effective theory, the $\delta$ functions at $y=0$ represent the contributions from the so-called zero modes on the light front. These are essential in order to ensure that fundamental symmetries, such as Lorentz invariance, chiral symmetry, and local gauge symmetry, are respected in the calculation. Experimentally, the physical appearance of this contribution at $x=0$ is still an open question. One may imagine that in more elaborate treatments that better capture the short-range structure of the intermediate state baryon in QCD, the $\delta$ function contribution may soften into a peak near $x=0$ [34]. However, in the absence of a solution to QCD in the strong coupling regime this remains speculative, and in the present formulation of the effective theory the $\delta$-function terms are a natural consequence.

For the $\pi \Delta$ contributions to the asymmetry in Eq. (45), the splitting function for the rainbow diagram in Fig. 1(b) includes several regular and $\delta$-function terms,

$$
\begin{aligned}
f_{\pi^{-} \Delta^{++}}^{(\mathrm{rbw})}(y)= & 3 f_{\pi^{+} \Delta^{0}}^{(\mathrm{rbw})}(y) \\
= & \frac{\mathcal{C}^{2} \bar{M}^{2}}{2(4 \pi f)^{2}}\left[f_{\Delta}^{(\mathrm{on})}(y)+f_{\Delta}^{(\mathrm{on} \mathrm{end})}(y)-\frac{1}{18} f_{\Delta}^{(\delta)}(y)\right. \\
& \left.+\frac{2 M^{2}\left(\bar{M}^{2}-m_{\pi}^{2}\right)}{3 M_{\Delta}^{2} \bar{M}^{2}}\left(f_{\pi}^{(\delta)}(y)-\delta f_{\pi}^{(\delta)}(y)\right)\right]
\end{aligned}
$$

where $\bar{M}=M+M_{\Delta}$ and $\mathcal{C}$ is the meson-octet-decuplet baryon coupling, which is related to the $\pi N \Delta$ coupling constant $g_{\pi N \Delta}$ by $\mathcal{C}=\sqrt{2} f g_{\pi N \Delta}[20,35]$. As for the $\pi N$ case, the on-shell function for the $\Delta$ intermediate state, $f_{\Delta}^{(\text {on })}$, is nonzero for $y>0$, with a shape that is qualitatively similar to $f_{N}^{\text {(on) }}$, but peaking at smaller $y$ because of the positive $\Delta$-nucleon mass difference $[20,36,37]$. The onshell end-point function, $f_{\Delta}^{\text {(on end) }}$, also has a similar shape for finite $\Lambda$, but in the $\Lambda \rightarrow \infty$ limit is associated with an end-point singularity that gives a $\delta$ function at $y=1$. The off-shell components of the $\Delta$ propagator induce several terms that are proportional to $\delta$ functions at $y=0$. The functions $f_{\pi}^{(\delta)}$ and $\delta f_{\pi}^{(\delta)}$ are equivalent to those in Eq. (46), while $f_{\Delta}^{(\delta)}$ is a new function that appears only for the decuplet intermediate state [35].
Finally, the bubble diagram contribution to the $\bar{d}-\bar{u}$ asymmetry, $f_{\pi}^{(\text {bub })}$, in Fig. $1(\mathrm{c})$ is given by the same combination of basis $\delta$-function contributions as for the rainbow diagrams,

$$
f_{\pi}^{(\mathrm{bub})}(y)=-\frac{2 M^{2}}{(4 \pi f)^{2}}\left[f_{\pi}^{(\delta)}(y)-\delta f_{\pi}^{(\delta)}(y)\right] .
$$

Although this term gives a nonzero PDF only at $x=0$, since it contributes to the integral of $\bar{d}-\bar{u}$, it will indirectly affect the normalization for $x>0$. On the other hand, experimental cross sections are in practice available only for $x>0$, so that the $\delta$-function pieces are generally difficult to constrain directly, especially in regularization schemes that use different regulator parameters for the $\delta$ function and $y>0$ contributions [35]. The advantage of the nonlocal approach employed here is that by consistently introducing a vertex form factor in coordinate space in the nonlocal Lagrangian [20], the same regulator function then appears in all splitting functions derived from the fundamental interaction, which in our case is parametrized through the single cutoff $\Lambda$. Even if experimental data constrain only contributions at $x>0$, such as from the onshell functions, once determined these can then be used to compute other contributions, including those at $x=0$.

Following Refs. [24,25,36], we can constrain the parameter $\Lambda$ for the octet intermediate states by comparing the one-pion exchange contribution with the differential cross section for the inclusive charge-exchange process $p p \rightarrow$ $n X$ at $y>0$,

$$
\sigma(p p \rightarrow n X)=\frac{2(D+F)^{2} M^{2}}{(4 \pi f)^{2}} \frac{\bar{y}}{\pi} \hat{f}_{N}^{(\mathrm{on})}\left(y, k_{\perp}^{2}\right) \sigma_{\mathrm{tot}}^{\pi^{+} p}(y s),
$$

where $s$ is the invariant mass squared of the reaction. The function $\hat{f}_{N}^{(\text {on })}\left(y, k_{\perp}^{2}\right)$ in Eq. (49) is the unintegrated on-shell nucleon splitting function, which is related to the corresponding integrated splitting function $f_{N}^{\text {(on) }}(y)$ in Eq. (46) by [see also Eq. (63) in Ref. [20]]

$$
f_{N}^{(\mathrm{on})}(y) \equiv \int d k_{\perp}^{2} \hat{f}_{N}^{\text {(on })}\left(y, k_{\perp}^{2}\right)
$$

The cross section $\sigma_{\text {tot }}^{\pi^{+} p}(y s)$ in Eq. (49) is the total $\pi^{+} p$ scattering cross section evaluated at the center of mass energy ys. In the numerical calculations, we use the (approximately energy independent) empirical value $\sigma_{\text {tot }}^{\pi^{+} p}=23.8(1) \mathrm{mb}$ [53]. For the SU(3) couplings we take $D=0.85$ and $F=0.41$, which gives a triplet axial charge $g_{A}=\left(2 \bar{\alpha}^{(1)}-\bar{\beta}^{(1)}\right) / 3=D+F=1.26$ and an octet axial charge $g_{8}=\bar{\alpha}^{(1)}+\bar{\beta}^{(1)}=3 F-D=0.38$. 

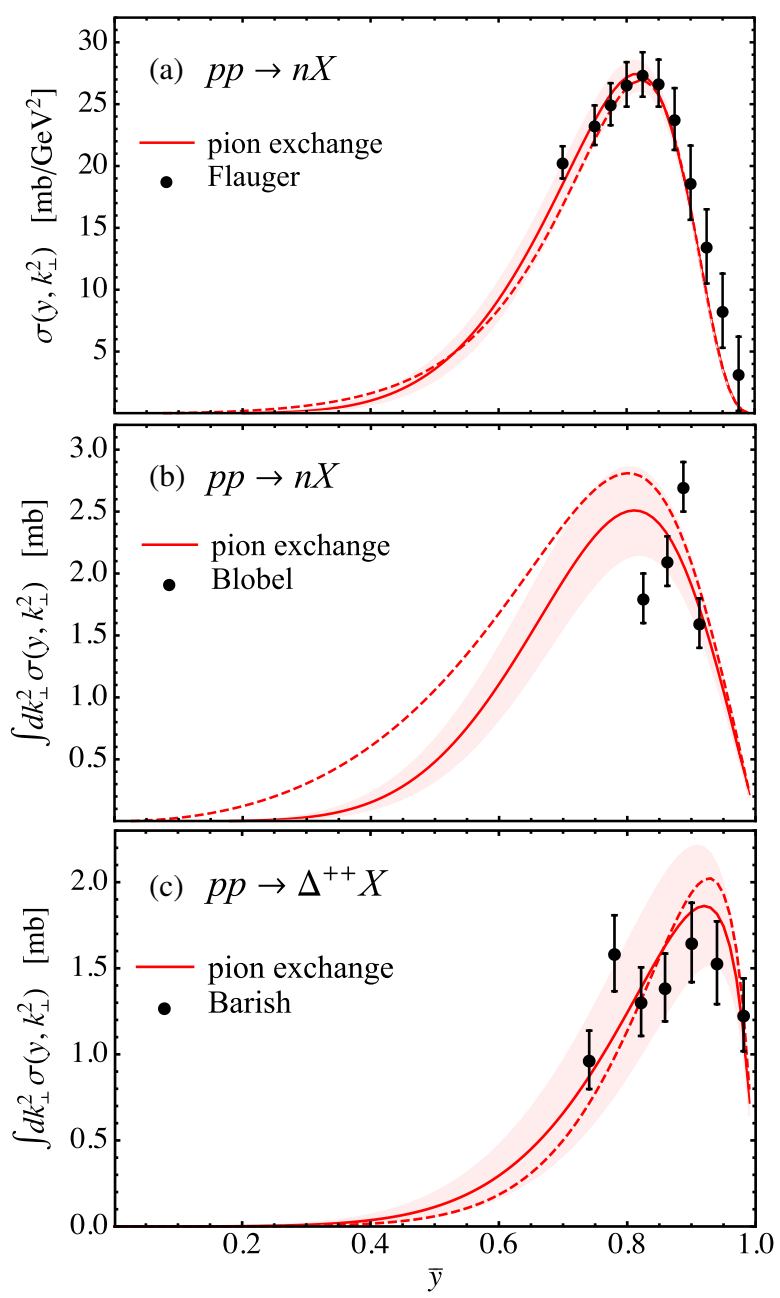

FIG. 3. Differential inclusive hadron production cross section $\sigma\left(y, k_{\perp}^{2}\right)$ vs $\bar{y}$ for (a) $p p \rightarrow n X$ at $k_{\perp}^{2}=0$ [38]; (b) $p p \rightarrow n X$ integrated over $k_{\perp}^{2}$ [39]; (c) $p p \rightarrow \Delta^{++} X$ integrated over $k_{\perp}^{2}$ [40], compared with the fitted nonlocal pion exchange contributions for $\Lambda_{\pi N}=1.0(1) \mathrm{GeV}$ and $\Lambda_{\pi \Delta}=0.9(1) \mathrm{GeV}$ (solid red lines and pink $1 \sigma$ uncertainty bands) and with Pauli-Villars regularization (dashed red lines) for $\Lambda_{\pi N}^{(\mathrm{PV})}=0.3 \mathrm{GeV}$ and $\Lambda_{\pi \Delta}^{(\mathrm{PV})}=0.64 \mathrm{GeV}$.

The results for the differential neutron production cross section are shown in Fig. 3 vs $\bar{y}$. The experimental data are typically presented as a function of the ratio $2 p_{L} / \sqrt{s}$, where $p_{L}$ is the longitudinal momentum of the produced baryon in the center of mass frame; at high energies, however, this is equivalent to $\bar{y}$. In Fig. 3(a) we compare our results with the neutron production data from the ISR at CERN at energies $\sqrt{s}$ between $\approx 31$ and $63 \mathrm{GeV}$ for $0^{\circ}$ neutron production angles, or $k_{\perp}^{2}=0$ [38]. Data from the hydrogen bubble chamber experiment at the CERN proton synchrotron at $\sqrt{s} \approx 5$ and $7 \mathrm{GeV}$ [39] are shown in Fig. 3(b) for the $k_{\perp}$-integrated neutron cross section. Because the pion-exchange process is dominant only at large $\bar{y}$ [54], with contributions from background processes such as the exchange of heavier mesons $[36,55,56]$ becoming more important at lower $\bar{y}$, we include data only in the region $\bar{y}>0.7$. Corrections from rescattering and absorption are also known to play a role in inclusive hadron production, and are estimated to be around $20 \%$ at high values of $\bar{y}$ [56-58]. A good description of the single and double differential neutron data can be achieved with a cutoff parameter $\Lambda_{\pi N}=1.0(1) \mathrm{GeV}$. A marginally larger value is found if fitting only the double differential data, and slightly smaller value for just the $k_{\perp}$-integrated cross section, but consistent within the uncertainties

For the inclusive production of decuplet baryons, the invariant differential cross section for an inclusive $\Delta^{++}$in the final state can be written for $y>0$ as

$$
\begin{aligned}
& \sigma\left(p p \rightarrow \Delta^{++} X\right) \\
& =\frac{\mathcal{C}^{2} \bar{M}^{2}}{2(4 \pi f)^{2}} \frac{\bar{y}}{\pi}\left[\hat{f}_{\Delta}^{\text {on })}\left(y, k_{\perp}^{2}\right)+\hat{f}_{\Delta}^{(\text {on end })}\left(y, k_{\perp}^{2}\right)\right] \sigma_{\text {tot }}^{\pi^{-} p}(y s),
\end{aligned}
$$

where $\sigma_{\mathrm{tot}}^{p \pi^{-}}$is the total $\pi^{-} p$ scattering cross section. In our numerical calculations we assume this to be charge independent, so that $\sigma_{\text {tot }}^{\pi^{-} p} \approx \sigma_{\text {tot }}^{\pi^{+} p}$, and for the coupling constant $\mathcal{C}$ we take the $\mathrm{SU}(6)$ symmetric value $\mathcal{C}=-2 D=$ -1.72 . The functions $\hat{f}_{\Delta}^{\text {(on })}\left(y, k_{\perp}^{2}\right)$ and $\hat{f}_{\Delta}^{\text {(on end })}\left(y, k_{\perp}^{2}\right)$ in (51) are the unintegrated decuplet on-shell and on-shell end-point splitting functions, which are related to the corresponding integrated splitting functions [see Eqs. (86)(88) in [20]] by the identities

$$
\begin{gathered}
f_{\Delta}^{\text {(on })}(y) \equiv \int d k_{\perp}^{2} \hat{f}_{\Delta}^{\text {(on })}\left(y, k_{\perp}^{2}\right), \\
f_{\Delta}^{\text {(on end })}(y) \equiv \int d k_{\perp}^{2} \hat{f}_{\Delta}^{\text {(on end })}\left(y, k_{\perp}^{2}\right),
\end{gathered}
$$

respectively. The $k_{\perp}^{2}$-integrated $\Delta^{++}$cross section is shown in Fig. 3(c) compared with hydrogen bubble chamber data taken at Fermilab for $\sqrt{s} \approx 20 \mathrm{GeV}$ [40]. A good fit to the data is obtained with a value of the decuplet cutoff of $\Lambda_{\pi \Delta}=0.9(1) \mathrm{GeV}$, which is slightly smaller than that for the neutron production cross sections.

To examine the model dependence of the analysis, for comparison we also fitted the hadron production cross sections in Figs. 3(a)-3(c) using instead the Pauli-Villars regularization for the local effective theory $[24,25]$. The explicit forms of the Pauli-Villars regularized octet on-shell splitting functions can be found in [24,25]. The result for the sum of the decuplet on-shell and on-shell end-point functions is as in Eq. (96) of [20], with the integral regularized by a factor of $\left(1+4 D_{\pi \Delta} / D_{\Lambda \Delta}\right)$, where $D_{\pi \Delta}$ and $D_{\Lambda \Delta}$ are momentum dependent functions given in Eq. (86) of [20]. The results for the best fit PauliVillars mass parameters $\Lambda_{\pi N}^{(\mathrm{PV})}=0.30 \mathrm{GeV}$ and $\Lambda_{\pi \Delta}^{(\mathrm{PV})}=$ $0.64 \mathrm{GeV}$ are illustrated by the dashed curves in Fig. 3 and 


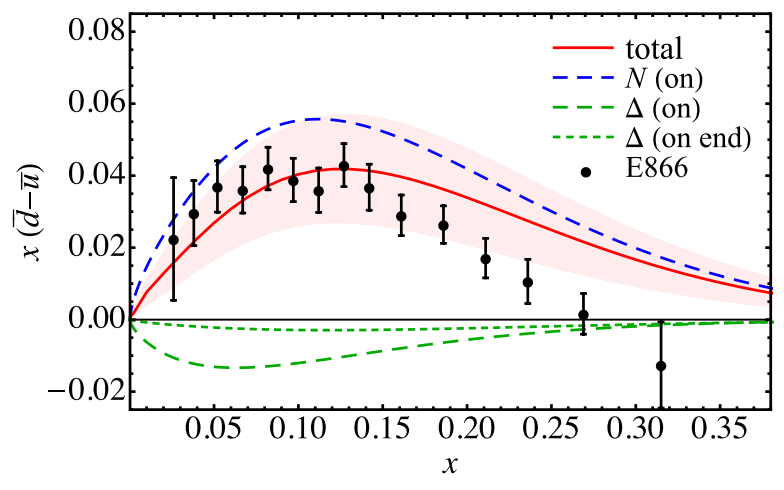

FIG. 4. The flavor asymmetry of the proton $x(\bar{d}-\bar{u})$ vs $x$ from the lowest order pion exchange (solid red curve and pink band), with cutoff parameters $\Lambda_{\pi N}=1.0(1) \mathrm{GeV}$ and $\Lambda_{\pi \Delta}=0.9(1) \mathrm{GeV}$, including nucleon on-shell (dashed blue line), $\Delta$ on-shell (dashed green line), and $\Delta$ end-point (dotted green line) contributions, and compared with the asymmetry extracted from the Fermilab E615 Drell-Yan experiment [4].

are similar to those for the nonlocal calculation, even though the numerical values for the cutoffs are rather different. While there is some difference in the shape of the calculated $k_{\perp}$-integrated neutron production cross section in Fig. 3(b) at smaller values of $\bar{y}$, in the region where the data provide constraints the Pauli-Villars results lie within the uncertainty bands of the nonlocal calculation using with the dipole regulator.

Using the values of $\Lambda_{\pi N}$ and $\Lambda_{\pi \Delta}$ for our nonlocal calculation constrained by the $p p$ cross sections in Fig. 3, we next evaluate the flavor asymmetry $\bar{d}-\bar{u}$ from the convolution of the splitting functions and the pion PDF in Eq. (45). The results for $x(\bar{d}-\bar{u})$ are shown in Fig. 4 and compared with the asymmetry extracted from the E866 Drell-Yan lepton-pair production data from Fermilab [4]. At nonzero $x$ values only the on-shell nucleon and $\Delta$ and end-point $\Delta$ terms contribute to the asymmetry, each of which is indicated in Fig. 4. The positive nucleon on-shell term makes the largest contribution, which is partially canceled by the negative $\Delta$ contributions. For the values of the cutoffs used here, the end-point term is relatively small compared with the on-shell $\Delta$ component.

Although the $\delta$-function contributions to the flavor asymmetry are not directly visible in Fig. 4, their effect can be seen in the lowest moment of the asymmetry,

$$
\langle\bar{d}-\bar{u}\rangle \equiv \int_{0}^{1} d x(\bar{d}(x)-\bar{u}(x)) .
$$

The contributions from the individual on-shell, end-point, and $\delta$-function components of the $\pi N$ and $\pi \Delta$ rainbow and the $\pi$ bubble diagrams to the moment are shown in Fig. 5 vs the dipole cutoff parameter $\Lambda\left(=\Lambda_{\pi N}\right.$ or $\left.\Lambda_{\pi \Delta}\right)$, for the approximate ranges of values found in the fits in Fig. 3. For the best fit values $\Lambda_{\pi N}=1.0(1) \mathrm{GeV}$ and $\Lambda_{\pi \Delta}=0.9(1) \mathrm{GeV}$, the contributions from the individual
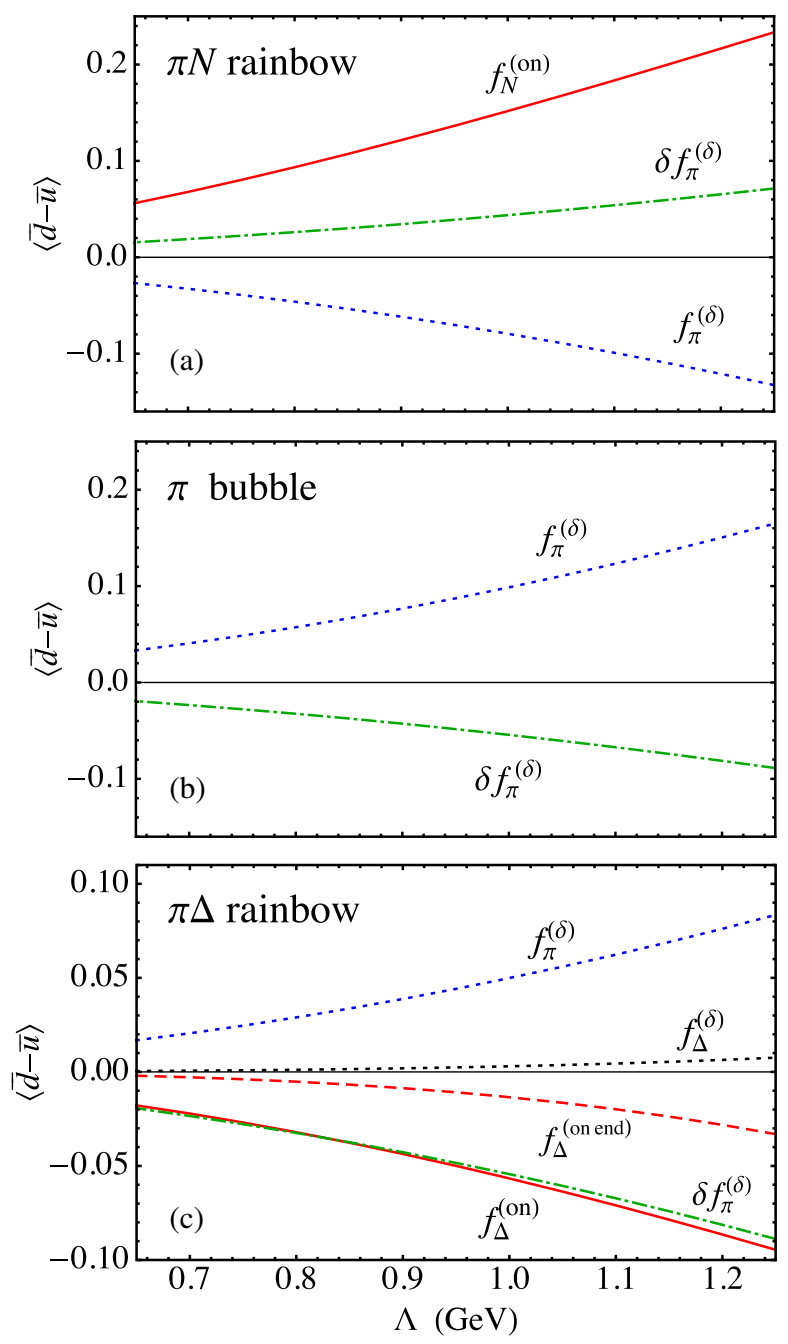

FIG. 5. Contributions to the $\langle\bar{d}-\bar{u}\rangle$ moment vs the dipole cutoff parameter $\Lambda$ ( $=\Lambda_{\pi N}$ or $\left.\Lambda_{\pi \Delta}\right)$ from (a) the $\pi N$ rainbow diagram [Fig. 1(a)], including on-shell (solid red line), and local (dotted blue line) and nonlocal (dot-dashed green line) $\delta$-function terms; (b) the pion bubble [Fig. 1(c)], including local (dotted blue line) and nonlocal (dot-dashed green line) $\delta$-function pieces; (c) the $\pi \Delta$ rainbow [Fig. 1(b)], including on-shell (solid red line), end-point (dashed red line), local (dotted blue line) and nonlocal (dot-dashed green line) $\delta$-function, and local decuplet $\delta$-function (dotted black line) contributions.

terms in Eqs. (45)-(48) are listed in Table VII, along with the combined contributions from the $x>0$ and $x=0$ terms, and the local and nonlocal terms, to the total integrated result. The nucleon on-shell term is the most important component, with a contribution that is within $\approx 20 \%$ of the total integrated value $\langle\bar{d}-\bar{u}\rangle=0.127_{-0.042}^{+0.044}$, where the errors reflect the uncertainties on the cutoff parameters. The on-shell and end-point $\pi \Delta$ terms yield overall negative contributions, with magnitude $\approx 30 \%$ of the on-shell $\pi N$. Furthermore, the breakdown into the local and nonlocal pieces shows that the latter is negative, with magnitude $\approx 20 \%$ of the local. 
TABLE VII. Contributions to the integral $\langle\bar{d}-\bar{u}\rangle \equiv \int_{0}^{1} d x(\bar{d}-\bar{u})$ from the $\pi N$ rainbow, $\pi \Delta$ rainbow, and $\pi$ bubble diagrams in Fig. 1, for the best fit parameters $\Lambda_{\pi N}=1.0(1) \mathrm{GeV}$ and $\Lambda_{\pi \Delta}=0.9(1) \mathrm{GeV}$. The contributions from the various terms in Eqs. (45)-(48) are listed individually, as are the combined contributions from $x>0$ and $x=0$, and the local and nonlocal terms, to the total. Note that some numbers do not sum to the totals because of rounding.

\begin{tabular}{lcc}
\hline \hline Diagram & & $\langle\bar{d}-\bar{u}\rangle$ \\
\hline$\pi N$ (rbw) & $f_{N}^{(\text {on })}$ & $0.152_{-0.030}^{+0.032}$ \\
& $f_{\pi}^{(\delta)}$ & $-\left(0.079_{-0.018}^{+0.020}\right)$ \\
& $\delta f_{\pi}^{(\delta)}$ & $0.044_{-0.009}^{+0.010}$ \\
Total $\pi N$ & & $0.116_{-0.022}^{+0.022}$ \\
\hline$\pi \Delta$ (rbw) & $f_{\Delta}^{(\text {on })}$ & $-\left(0.044_{-0.012}^{+0.012}\right)$ \\
& $f_{\Delta}^{(\text {on end })}$ & $-\left(0.009_{-0.003}^{+0.004}\right)$ \\
& $f_{\Delta}^{(\delta)}$ & $0.002_{-0.001}^{+0.001}$ \\
Total $\pi \Delta$ & $f_{\pi}^{(\delta)}$ & $0.039_{-0.010}^{+0.010}$ \\
\hline$\pi$ (bub) & $\delta f_{\pi}^{(\delta)}$ & $-\left(0.022_{-0.005}^{+0.005}\right)$ \\
Total $\pi$ bubble & & $-\left(0.033_{-0.010}^{+0.010}\right)$ \\
\hline Total & $f_{\pi}^{(\delta)}$ & $0.099_{-0.022}^{+0.025}$ \\
\hline \hline$x>0$ & $\delta f_{\pi}^{(\delta)}$ & $-\left(0.054_{-0.012}^{+0.013}\right)$ \\
$x=0$ & & $0.044_{-0.010}^{+0.012}$ \\
\hline Local & & $\mathbf{0 . 1 2 7 _ { - 0 . 0 4 2 } ^ { + 0 . 0 4 4 }}$ \\
\hline \hline Nonlocal & & $0.099_{-0.046}^{+0.047}$ \\
& & $0.028_{-0.007}^{+0.008}$ \\
\hline
\end{tabular}

The various $\delta$-function terms from all three diagrams in Fig. 1 cancel to a considerable degree, with the $x=0$ contribution making up $\approx 20 \%$ of the total. This contribution is smaller than the total uncertainty due to the variation of the cutoff parameter $\Lambda$, making it difficult to draw unambiguous conclusions about the phenomenological role of the $\delta$-function terms. With more precise experimental data on the inclusive $p p$ baryon production cross sections and better theoretical constraints on our model, the range of $\Lambda$ could be further reduced. Eventually, if the total uncertainty on $\Lambda$ is smaller than the $\delta$-function contributions, one could hope to make firmer conclusions about the $x=0$ terms.

Experimentally, the asymmetry at $x=0$ is of course not directly measurable, and typically extrapolations are made to estimate contributions from outside of the measured region. The New Muon Collaboration, for instance, found $\langle\bar{d}-\bar{u}\rangle_{\mathrm{NMC}}^{(\exp )}=0.169(32)$ from their analysis of $F_{2}^{p}-F_{2}^{n}$ in the experimentally accessible region $0.004 \leq x \leq 0.8$, and
$\langle\bar{d}-\bar{u}\rangle_{\mathrm{NMC}}^{(\text {tot })}=0.148(39)$ when including $x \rightarrow 0$ and $x \rightarrow 1$ extrapolations [1]. The E866 Collaboration, on the other hand, extracted $\langle\bar{d}-\bar{u}\rangle_{\mathrm{E} 866}^{(\exp )}=0.080(11)$ in the experimentally measured interval $0.015 \leq x \leq 0.035$, and $\langle\bar{d}-\bar{u}\rangle_{\mathrm{E} 866}^{(\mathrm{tot})}=0.118(12)$ for the entire $x$ range after extrapolation. Note that the extrapolations by different analyses are often based on different assumptions for the asymptotic $x \rightarrow 0$ and $x \rightarrow 1$ behavior, so that a direct comparison of extrapolated results is problematic. Nevertheless, the general magnitude of the asymmetry is comparable with that found in our calculation, even with the uncertainties about the $x=0$ and extrapolated contributions.

\section{B. $s-\bar{s}$ asymmetry}

While the $\bar{d}-\bar{u}$ asymmetry is perhaps the best known consequence of pion loops on PDFs in the nucleon, an equally intriguing ramification of $\mathrm{SU}(3)$ chiral symmetry breaking is the $s-\bar{s}$ asymmetry generated by kaon loops. In analogy to the light antiquark PDFs in Eq. (45), the contribution to the antistrange PDF in the proton arising from kaon loops in Fig. 1 can be written as

$$
\bar{s}(x)=\left[\left(\sum_{\phi B} f_{\phi B}^{(\mathrm{rbw})}+\sum_{\phi T} f_{\phi T}^{(\mathrm{rbw})}+\sum_{\phi} f_{\phi}^{(\mathrm{bub})}\right) \otimes \bar{s}_{K}\right](x),
$$

where here the sums are over the states $\phi B=$ $\left\{K^{+} \Lambda, K^{+} \Sigma^{0}, K^{0} \Sigma^{+}\right\}$for the kaon-octet baryon rainbow diagram [Fig. 1(a)], $\phi T=\left\{K^{+} \Sigma^{* 0}, K^{0} \Sigma^{*+}\right\}$ for the kaondecuplet baryon rainbow diagram [Fig. 1(b)], and for the $\phi=K^{+}\left(K^{-}\right)$and $K^{0}\left(\bar{K}^{0}\right)$ loop in the bubble diagram [Fig. 1(c)]. In terms of the on-shell and $\delta$-function basis functions, the kaon-octet baryon rainbow function can be written is given by a form similar to that in Eq. (46),

$$
\begin{aligned}
f_{K^{+} \Lambda}^{\text {(rbw }}(y)= & \frac{(D+3 F)^{2}\left(M+M_{\Lambda}\right)}{12(4 \pi f)^{2}} \\
& \times\left[f_{\Lambda}^{(\mathrm{on})}(y)+f_{K}^{(\delta)}(y)-\delta f_{K}^{(\delta)}(y)\right], \\
2 f_{K^{+} \Sigma^{0}}^{(\mathrm{rbw})}(y)= & f_{K^{0} \Sigma^{+}}^{\text {(rbw) }}(y)=\frac{(D-F)^{2}\left(M+M_{\Sigma}\right)}{2(4 \pi f)^{2}} \\
& \times\left[f_{\Sigma}^{\text {(on })}(y)+f_{K}^{(\delta)}(y)-\delta f_{K}^{(\delta)}(y)\right],
\end{aligned}
$$

for the $K \Lambda$ and $K \Sigma$ intermediate states, respectively. For the kaon-decuplet baryon rainbow diagram, the corresponding function is written analogously to Eq. (47), 


$$
\begin{aligned}
2 f_{K^{+} \Sigma^{* 0}}^{(\mathrm{rbw})}(y)= & f_{K^{0} \Sigma^{*+}}^{(\mathrm{rbw})}(y)=\frac{\mathcal{C}^{2}\left(M+M_{\Sigma^{*}}\right)^{2}}{6(4 \pi f)^{2}} \\
& \times\left[f_{\Sigma^{*}}^{\text {(on })}(y)+f_{\Sigma^{*}}^{\text {on end })}(y)-\frac{1}{18} f_{\Sigma^{*}}^{(\delta)}(y)\right. \\
& +\frac{\left(M+M_{\Sigma}\right)^{2}\left[\left(M+M_{\Sigma^{*}}\right)^{2}-m_{K}^{2}\right]}{6 M_{\Sigma^{*}}^{2}\left(M+M_{\Sigma^{*}}\right)^{2}} \\
& \left.\times\left(f_{K}^{(\delta)}(y)-\delta f_{K}^{(\delta)}(y)\right)\right]
\end{aligned}
$$

for the $K \Sigma^{*}$ states, where the coupling $\mathcal{C}$ is given in the previous section. For the bubble diagram, the splitting function for charged or neutral kaon loops is given by a form similar to that in Eq. (48),

$f_{K^{+}}^{(\mathrm{bub})}(y)=2 f_{K^{0}}^{(\mathrm{bub})}(y)=-\frac{\left(M+M_{\Sigma}\right)^{2}}{(4 \pi f)^{2}}\left[f_{K}^{(\delta)}(y)-\delta f_{K}^{(\delta)}(y)\right]$.

Explicit expressions for all the basis functions are given in Ref. [20].

For the loop contributions to the strange quark PDF, the baryon-coupling rainbow, Kroll-Ruderman, and tadpole diagrams in Figs. 2(d)-2(k) all play a role, as do the additional gauge-link dependent diagrams that are generated by the nonlocal Lagrangian. Assuming that all nonperturbatively generated strangeness resides in the intermediate state hyperons, from Eq. (10) the loop contributions to the strange quark PDF in the proton can be written as

$$
\begin{aligned}
s(x)= & \sum_{B \phi}\left\{\left[\bar{f}_{B \phi}^{(\mathrm{rbw})} \otimes s_{B}\right](x)+\left[\bar{f}_{B}^{(\mathrm{KR})} \otimes s_{B}^{(\mathrm{KR})}\right](x)\right. \\
& \left.+\left[\delta \bar{f}_{B}^{(\mathrm{KR})} \otimes s_{B}^{(\delta)}\right](x)\right\}+\sum_{T \phi}\left\{\left[\bar{f}_{T \phi}^{(\mathrm{rbw})} \otimes s_{T}\right](x)\right. \\
& \left.+\left[\bar{f}_{T}^{(\mathrm{KR})} \otimes s_{T}^{(\mathrm{KR})}\right](x)+\left[\delta \bar{f}_{T}^{(\mathrm{KR})} \otimes s_{T}^{(\delta)}\right](x)\right\} \\
& +\sum_{\phi}\left\{\left[\bar{f}_{\phi}^{(\mathrm{tad})} \otimes s_{\phi}^{(\mathrm{tad})}\right](x)+\left[\delta \bar{f}_{\phi}^{(\mathrm{tad})} \otimes s_{\phi}^{(\delta)}\right](x)\right\},
\end{aligned}
$$

where the sums are over the octet bayon-meson states $B \phi=\left\{\Lambda K^{+}, \Sigma^{0} K^{+}, \Sigma^{+} K^{0}\right\}$, decuplet baryon-meson states $T \phi=\left\{\Sigma^{* 0} K^{+}, \Sigma^{*+} K^{0}\right\}$, and mesons $\phi=K^{+}\left(K^{-}\right)$and $K^{0}\left(\bar{K}^{0}\right)$ for the tadpole contributions. As in Eq. (10), the splitting functions for all the hyperon coupling diagrams in Eq. (58) use the shorthand notation $\bar{f}_{j}(y) \equiv f_{j}(1-y)$.

For the octet hyperon rainbow diagrams, Fig. 2(d), the individual splitting functions can be written in terms of the on-shell, off-shell, and $\delta$-function basis functions as

$$
\begin{aligned}
f_{\Lambda K^{+}}^{(\mathrm{rbw})}(y)= & \frac{(D+3 F)^{2}\left(M+M_{\Lambda}\right)^{2}}{12(4 \pi f)^{2}} \\
& \times\left[f_{\Lambda}^{\text {(on })}(y)+f_{\Lambda}^{(\mathrm{off})}(y)+4 \delta f_{\Lambda}^{(\mathrm{off})}(y)-f_{K}^{(\delta)}(y)\right], \\
2 f_{\Sigma^{0} K^{+}}^{(\mathrm{rbw})}(y)= & f_{\Sigma^{+} K^{0}}^{\text {(rbw) }}(y)=\frac{(D-F)^{2}\left(M+M_{\Sigma}\right)^{2}}{2(4 \pi f)^{2}} \\
& \times\left[f_{\Sigma}^{\text {(on })}(y)+f_{\Sigma}^{\text {(off })}(y)+4 \delta f_{\Sigma}^{\text {(off })}(y)-f_{K}^{(\delta)}(y)\right],
\end{aligned}
$$

where the functions $f_{\Lambda, \Sigma}^{(\text {on })}$ and $f_{K}^{(\delta)}$ are the same as in Eq. (55), and explicit expressions for the off-shell functions $f_{\Lambda, \Sigma}^{\text {(off) }}$ and $\delta f_{\Lambda, \Sigma}^{\text {(off) }}$ are given in Sec. IV. B. 1 of Ref. [20]. For the octet Kroll-Ruderman diagrams in Figs. 2(e) and 2(f), the local and nonlocal splitting functions $f_{\Lambda, \Sigma}^{(\mathrm{KR})}$ and $\delta f_{\Lambda, \Sigma}^{(\mathrm{KR})}$ are given by

$$
f_{\Lambda}^{(\mathrm{KR})}(y)=\frac{(D+3 F)^{2}\left(M+M_{\Lambda}\right)^{2}}{12(4 \pi f)^{2}}\left[-f_{\Lambda}^{(\mathrm{off})}(y)+2 f_{K}^{(\delta)}(y)\right],
$$

$$
\begin{aligned}
2 f_{\Sigma^{0}}^{(\mathrm{KR})}(y) & =f_{\Sigma^{+}}^{(\mathrm{KR})}(y) \\
& =\frac{(D-F)^{2}\left(M+M_{\Sigma}\right)^{2}}{2(4 \pi f)^{2}}\left[-f_{\Sigma}^{(\mathrm{off})}(y)+2 f_{K}^{(\delta)}(y)\right],
\end{aligned}
$$

and

$$
\begin{aligned}
\delta f_{\Lambda}^{(\mathrm{KR})}(y) & =\frac{(D+3 F)^{2}\left(M+M_{\Lambda}\right)^{2}}{12(4 \pi f)^{2}}\left[-4 \delta f_{\Lambda}^{(\mathrm{off})}(y)-\delta f_{K}^{(\delta)}(y)\right], \\
2 \delta f_{\Sigma^{0}}^{(\mathrm{KR})}(y) & =\delta f_{\Sigma^{+}}^{(\mathrm{KR})}(y) \\
& =\frac{(D-F)^{2}\left(M+M_{\Sigma}\right)^{2}}{2(4 \pi f)^{2}}\left[-4 \delta f_{\Sigma}^{(\text {off })}(y)-\delta f_{K}^{(\delta)}(y)\right],
\end{aligned}
$$

respectively.

For the decuplet hyperon contributions, the respective splitting functions are given by

$$
\begin{aligned}
2 f_{\Sigma^{* 0} K^{+}}^{(\mathrm{rbw})}(y)= & f_{\Sigma^{*+} K^{0}}^{(\mathrm{rbw})}(y)=\frac{\mathcal{C}^{2}\left(M+M_{\Sigma^{*}}\right)^{2}}{6(4 \pi f)^{2}} \times\left[f_{\Sigma^{*}}^{(\text {on })}(y)+f_{\Sigma^{*}}^{(\text {on end })}(y)-2 f_{\Sigma^{*}}^{(\text {off })}(y)-2 f_{\Sigma^{*}}^{(\text {off end })}(y)+4 \delta f_{\Sigma^{*}}^{(\text {off })}(y)+\frac{1}{18} f_{\Sigma^{*}}^{(\delta)}(y)\right. \\
& \left.-\frac{1}{6} \delta f_{\Sigma^{*}}^{(\delta)}(y)-\frac{\left(M+M_{\Sigma}\right)^{2}\left[\left(M+M_{\Sigma^{*}}\right)^{2}+3 m_{K}^{2}\right]}{6 M_{\Sigma^{*}}^{2}\left(M+M_{\Sigma^{*}}\right)^{2}} f_{K}^{(\delta)}(y)\right]
\end{aligned}
$$


for the decuplet rainbow diagram in Fig. 2(g),

$$
\begin{aligned}
2 f_{\Sigma^{* 0}}^{(\mathrm{KR})}(y)= & f_{\Sigma^{*+}}^{(\mathrm{KR})}(y) \\
= & \frac{\mathcal{C}^{2}\left(M+M_{\Sigma^{*}}\right)^{2}}{6(4 \pi f)^{2}}\left[2 f_{\Sigma^{*}}^{(\mathrm{off})}(y)+2 f_{\Sigma^{*}}^{(\mathrm{off} \text { end })}(y)\right. \\
& -\frac{1}{9}\left(f_{\Sigma^{*}}^{(\delta)}(y)-\delta f_{\Sigma^{*}}^{(\delta)}(y)\right) \\
& \left.+\frac{\left(M+M_{\Sigma}\right)^{2}\left[\left(M+M_{\Sigma^{*}}\right)^{2}+m_{K}^{2}\right]}{3 M_{\Sigma^{*}}^{2}\left(M+M_{\Sigma^{*}}\right)^{2}} f_{K}^{(\delta)}(y)\right]
\end{aligned}
$$

for the Kroll-Ruderman diagram in Fig. 2(h), and

$$
\begin{aligned}
2 \delta f_{\Sigma^{* 0}}^{(\mathrm{KR})}(y)= & \delta f_{\Sigma^{*+}}^{(\mathrm{KR})}(y) \\
= & \frac{\mathcal{C}^{2}\left(M+M_{\Sigma^{*}}\right)^{2}}{6(4 \pi f)^{2}}\left[-4 \delta f_{\Sigma^{*}}^{(\mathrm{off})}(y)+\frac{1}{18} \delta f_{\Sigma^{*}}^{(\delta)}(y)\right. \\
& \left.-\frac{\left(M+M_{\Sigma}\right)^{2}\left[\left(M+M_{\Sigma^{*}}\right)^{2}-m_{K}^{2}\right]}{6 M_{\Sigma^{*}}^{2}\left(M+M_{\Sigma^{*}}\right)^{2}} \delta f_{K}^{(\delta)}(y)\right]
\end{aligned}
$$

for the nonlocal Kroll-Ruderman diagram in Fig. 2(i). The expressions for the decuplet basis functions $f_{\Sigma^{*}}^{(\text {on })}, f_{\Sigma^{*}}^{(\text {on end })}$, $f_{\Sigma^{*}}^{\text {(off) }}, f_{\Sigma^{*}}^{\text {(off end) }}$, and $f_{\Sigma^{*}}^{(\delta)}$, as well as the nonlocal functions $\delta f_{\Sigma^{*}}^{(\text {off })}$ and $\delta f_{\Sigma^{*}}^{(\delta)}$, are given in Sec. IV.B.2 of Ref. [20].

Finally, for the local and nonlocal tadpole contributions to the strange quark PDF from Figs. 2(j) and 2(k), the splitting functions are given by

$$
\begin{gathered}
f_{K^{+}}^{(\mathrm{tad})}(y)=2 f_{K^{0}}^{(\mathrm{tad})}(y)=-\frac{\left(M+M_{\Sigma}\right)^{2}}{(4 \pi f)^{2}} f_{K}^{(\delta)}(y), \\
\delta f_{K^{+}}^{(\mathrm{tad})}(y)=2 \delta f_{K^{0}}^{(\mathrm{tad})}(y)=\frac{\left(M+M_{\Sigma}\right)^{2}}{(4 \pi f)^{2}} \delta f_{K}^{(\delta)}(y),
\end{gathered}
$$

in terms of the local and nonlocal basis functions $f_{K}^{(\delta)}$ and $\delta f_{K}^{(\delta)}$.

To determine the regulator mass parameter for the kaonhyperon-nucleon vertices in Figs. 1 and 2, we consider inclusive hyperon production cross sections in $p p$ collisions, in analogy with the neutron and $\Delta$ production above. Data on inclusive $\Lambda$ production are available from the $2 \mathrm{~m}$ hydrogen bubble chamber at the CERN proton synchrotron [39] and the 12 foot hydrogen bubble chamber at ANL [41], and on inclusive $\Sigma^{*}$ production from CERN bubble chamber experiments [42]. The corresponding differential cross sections for inclusive $\Lambda$ and $\Sigma^{*}$ production (for $y>0$ ) are given by

$$
\begin{aligned}
& \sigma(p p \rightarrow \Lambda X) \\
& \quad=\frac{(D+3 F)^{2}\left(M+M_{\Lambda}\right)^{2}}{12(4 \pi f)^{2}} \frac{\bar{y}}{\pi} \hat{f}_{\Lambda}^{(\mathrm{on})}\left(y, k_{\perp}^{2}\right) \sigma_{\mathrm{tot}}^{K^{+} p}(y s),
\end{aligned}
$$

$$
\begin{aligned}
\sigma\left(p p \rightarrow \Sigma^{*+} X\right)= & \frac{\mathcal{C}^{2}\left(M+M_{\Sigma^{*}}\right)^{2}}{6(4 \pi f)^{2}} \frac{\bar{y}}{\pi}\left[\hat{f}_{\Sigma^{*+}}^{\text {oo })}\left(y, k_{\perp}^{2}\right)\right. \\
& \left.+\hat{f}_{\Sigma^{*+}}^{\text {(on end })}\left(y, k_{\perp}^{2}\right)\right] \sigma_{\text {tot }}^{K^{0} p}(y s),
\end{aligned}
$$

where $\hat{f}_{\Lambda}^{\text {(on })}, \hat{f}_{\Sigma^{*+}}^{\text {(on })}$, and $\hat{f}_{\Sigma^{*+}}^{\text {(on end })}$ are the $k_{\perp}$-unintegrated splitting functions defined from the on-shell and end-point basis functions [see Eqs. (63), (86), and (88) in Ref. [20]] by the relations

$$
\begin{aligned}
f_{\Lambda}^{\text {on })}(y) & \equiv \int d k_{\perp}^{2} \hat{f}_{\Lambda}^{\text {on })}\left(y, k_{\perp}^{2}\right), \\
f_{\Sigma^{*+}}^{(\text {on })}(y) & \equiv \int d k_{\perp}^{2} \hat{f}_{\Sigma^{*+}}^{\text {on })}\left(y, k_{\perp}^{2}\right), \\
f_{\Sigma^{*+}}^{\text {(on end })}(y) & \equiv \int d k_{\perp}^{2} \hat{f}_{\Sigma^{*+}}^{\text {on end })}\left(y, k_{\perp}^{2}\right) .
\end{aligned}
$$

In Eqs. (67) and (68) $\sigma_{\text {tot }}^{K^{+} p}$ and $\sigma_{\text {tot }}^{K^{0} p}$ are the total kaon-proton scattering cross sections, evaluated at invariant mass ys. For the numerical calculations we take the empirical value for $\sigma_{\text {tot }}^{K^{+} p}=19.9(1) \mathrm{mb}$ from Ref. [53], independent of energy. As there are no data for the $K^{0} p$ total cross section, we assume charge symmetry and relate this to the measured $K^{+} n$ cross section, $\sigma_{\text {tot }}^{K^{0} p} \approx \sigma_{\text {tot }}^{K^{+} n}=$ 19.7(1) mb [59].

In a similar vein to the pion exchange analysis of neutron and $\Delta$ production discussed above, in Fig. 6 we compare the inclusive $p p \rightarrow \Lambda X$ and $\Sigma^{*+} X$ cross sections for $\bar{y}>$ 0.7 with the kaon exchange contributions calculated from Eqs. (67) and (68). It has been established in several analyses $[53,54,56,58]$ that rescattering and absorption effects in inclusive baryon production result in $\lesssim 20 \%$ corrections at high $\bar{y}$. The previous studies found that the $t$ dependence at large values of $\bar{y}$ is consistent with that produced by meson exchange, and in our numerical analysis we follow the earlier work $[54,56,57]$ by focusing on the region $\bar{y}>0.7$.

The best fit to the CERN bubble chamber $\Lambda$ production data from Ref. [39] at $k_{\perp}=0.075 \mathrm{GeV}$ [Fig. 6(a)] and the $k_{\perp}$-integrated data from Ref. [41] [Fig. 6(b)] yields a dipole regulator mass $\Lambda_{K \Lambda}=1.1$ (1) GeV, similar to the value found for the $\pi N$ cutoff parameter from the inclusive neutron production data in Fig. 3. Comparison of the singly differential decuplet $\Sigma^{*+}$ production data at large $\bar{y}$ [Fig. 6(c)] with the kaon exchange cross section in Eq. (68) gives a best fit for the decuplet regulator mass of $\Lambda_{K \Sigma^{*}}=0.8(1) \mathrm{GeV}$. The cutoff parameter for the decuplet baryon is again slightly smaller than that for the octet baryon, as was found for the pion exchange contributions to the neutron and $\Delta$ cross sections in Fig. 3. Of course, the cutoff parameters could also in principle be constrained from data on kaon production, where the kaon is produced 

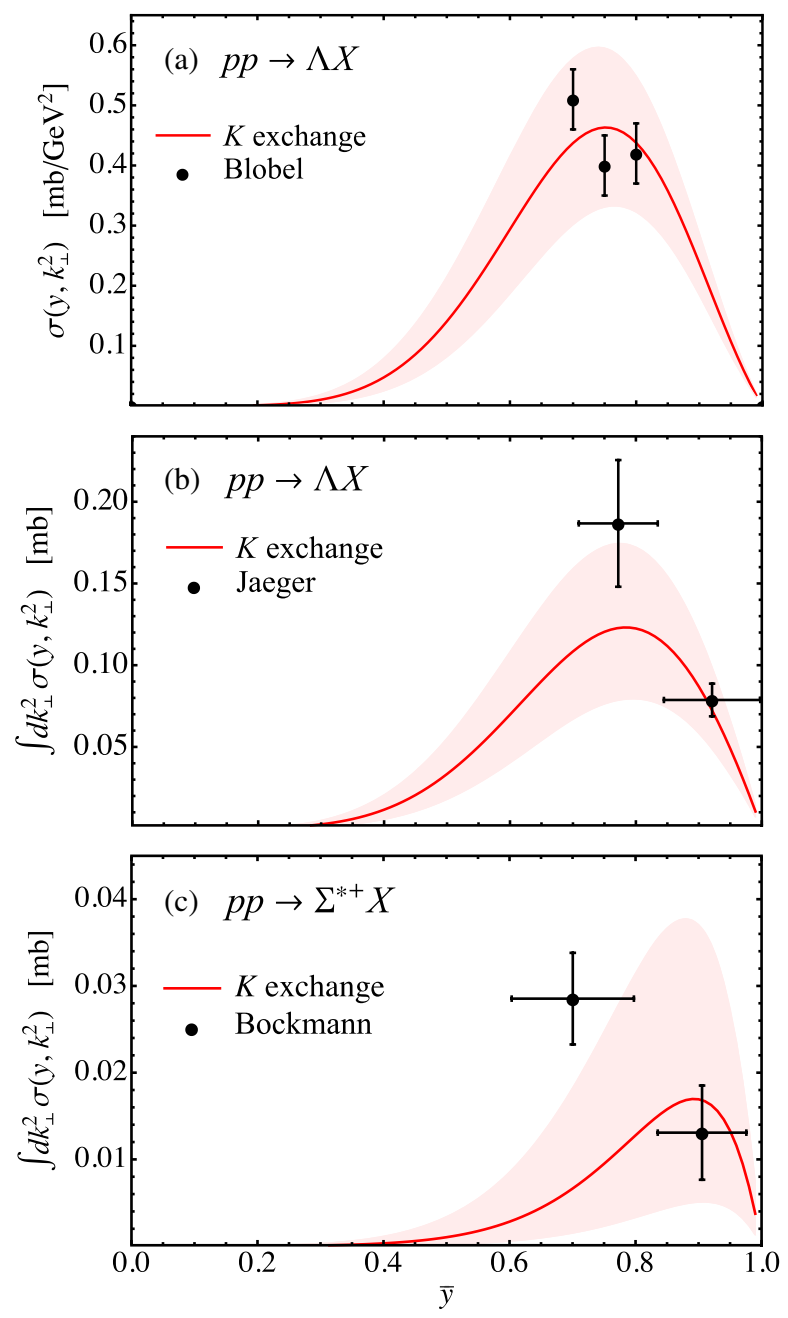

FIG. 6. Differential inclusive hadron production cross section $\sigma\left(y, k_{\perp}^{2}\right)$ vs $\bar{y}$ for (a) $p p \rightarrow \Lambda X$ at $k_{\perp}=0.075 \mathrm{GeV}$ [39]; (b) $p p \rightarrow \Lambda X$ integrated over $k_{\perp}^{2}$ [41]; and (c) $p p \rightarrow \Sigma^{*+} X$ integrated over $k_{\perp}^{2}$ [42], compared with the fitted nonlocal kaon exchange contributions for dipole regulator parameters $\Lambda_{K \Lambda}=$ 1.1(1) $\mathrm{GeV}$ and $\Lambda_{K \Sigma^{*}}=0.8(1) \mathrm{GeV}$ (solid red lines and pink $1 \sigma$ uncertainty bands).

in conjunction with scattering from an exchanged virtual baryon, as in Figs. 2(d) and 2(g). However, the backgrounds from kaons produced through quark fragmentation will be significant, making it considerably more difficult to identify mesons produced through $u$-channel baryon exchange and fragmentation.

With these values of the cutoffs, we can compute the kaon loop contributions to the strange and antistrange distributions in the proton, and estimate the shape and magnitude of the strange asymmetry $s-\bar{s}$. In Fig. 7 the various octet and decuplet contributions to $x s$ and $x \bar{s}$ are shown for the best fit parameters $\Lambda_{K \Lambda}=\Lambda_{K \Sigma}=1.1 \mathrm{GeV}$ and $\Lambda_{K \Sigma^{*}}=0.8 \mathrm{GeV}$. For the $x \bar{s}$ PDF in Fig. 7(a), the octet on-shell contribution from the rainbow diagram [Fig. 1(a)] dominates over the decuplet on-shell and end-point terms from the decuplet rainbow [Fig. 1(b)]. The resulting $x \bar{s}$ distribution peaks at $x \approx 0.1$ and essentially vanishes beyond $x \approx 0.6$. The $\delta$-function terms from the rainbow diagrams as well as from the kaon bubble diagram [Fig. 1(c)] contribute to $\bar{s}$ only at $x=0$ and so do not appear in Fig. 7(a).

In contrast, for the strange quark distribution, from the convolution in Eq. (58) one finds that all terms from each of the rainbow, Kroll-Ruderman, and tadpole diagrams in Figs. 2(d)-2(k) have nonzero contributions at $x>0$. Since there are many individual terms, we display ones involving octet + tadpole and decuplet baryons separately in Figs. 7(b) and 7(c), respectively. Unlike the on-shell term dominance of the antistrange PDF, for the strange distribution there are sizable contributions from many of the terms, with nontrivial cancellations between them. For the octet baryons, the off-shell terms change sign at around $x \approx 0.1$, with significant cancellation occurring between the local and nonlocal (gauge link dependent) off-shell contributions. The (positive) local and (negative) nonlocal $\delta$ function terms come with the largest magnitudes, but mostly cancel among themselves, leaving a total octet contribution that is positive and peaks around $x \approx 0.2$, with a similar order of magnitude as the $x \bar{s}$ distribution.

A qualitatively similar scenario is evident in Fig. 7(c) for the decuplet intermediate state contributions to $x s$, where the individual on-shell, off-shell, $\delta$-function, and gauge link terms are shown. (Note that the on-shell and off-shell terms include also the respective end-point pieces.) The predominantly positive on-shell, off-shell, and nonlocal $\delta$-function contributions at $x \gtrsim 0.2$ largely cancel with the predominantly negative local $\delta$-function and nonlocal off-shell terms, resulting in a very small overall decuplet contribution to $x s$, peaking at $x \sim 0.1$, that is, an order of magnitude smaller than the octet.

Finally, the resulting asymmetry $x(s-\bar{s})$ in Fig. 7(d) reflects the interplay between the $\bar{s}$ PDF, which peaks at lower $x$, and the $s$-quark PDF, which extends to larger values of $x$. A key feature of this result is the strong cancellations between positive local and negative nonlocal, gauge-link dependent contributions, in both the octet and the decuplet channels. The net effect is then a small positive $x(s-\bar{s})$ asymmetry, peaking at $x \approx 0.2-0.3$, and about an order of magnitude smaller than the asymmetry between the $\bar{d}$ and $\bar{u}$ PDFs resulting from pion loops.

In addition to the shape, it is instructive also to examine the contributions of the various terms to the lowest moments of the $s$ and $\bar{s}$ PDFs, in particular, the average number of strange and antistrange quarks,

$$
\langle s\rangle=\int_{0}^{1} d x s(x), \quad\langle\bar{s}\rangle=\int_{0}^{1} d x \bar{s}(x),
$$

and the average momentum carried by them, 

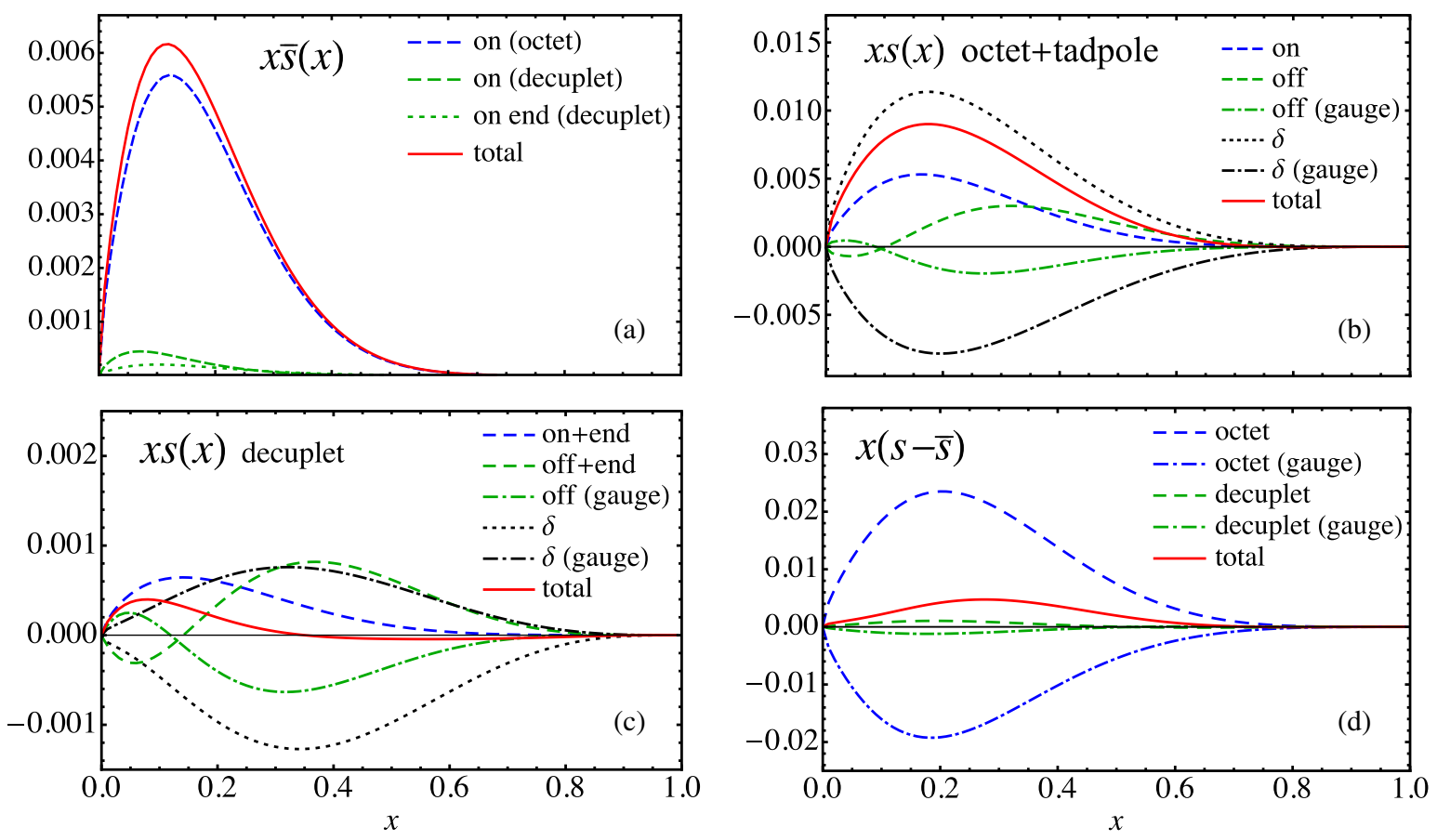

FIG. 7. Kaon loop contributions to (a) antistrange PDF $x \bar{s}$ from the octet and decuplet rainbow diagrams [Figs. 1(a) and 1(b)]; (b) strange quark PDF $x s$ from the octet rainbow [Fig. 2(d)], Kroll-Ruderman [Figs. 2(e) and 2(f)], and tadpole [Figs. 2(j) and 2(k)] diagrams; (c) strange PDF xs from the decuplet rainbow [Fig. 2(g)] and Kroll-Ruderman [Figs. 2(h) and 2(i)] diagrams; (d) strange asymmetry $x(s-\bar{s})$, showing the local and nonlocal (gauge) octet and decuplet contributions, along with the total asymmetry. The PDFs are computed with the best fit regulator parameters $\Lambda_{K \Lambda}=\Lambda_{K \Sigma}=1.1 \mathrm{GeV}$ and $\Lambda_{K \Sigma^{*}}=0.8 \mathrm{GeV}$.

$$
\langle x s\rangle=\int_{0}^{1} d x x s(x), \quad\langle x \bar{s}\rangle=\int_{0}^{1} d x x \bar{s}(x) .
$$

The conservation of strangeness of course requires equal numbers of $s$ and $\bar{s}$ quarks in the nucleon, $\langle s\rangle=\langle\bar{s}\rangle$, as a direct consequence of local gauge invariance, although the shapes of the $s$ and $\bar{s}$ distributions themselves are obviously rather different. The zero net strangeness can be verified by explicitly summing the contributions to $\langle s\rangle$ and $\langle\bar{s}\rangle$ from the various diagrams in Figs. 1 and 2, as Table VIII indicates. Note also that the conservation of strangeness holds for the octet and decuplet contributions individually, as well as for the tadpole and bubble diagrams,

$\langle s\rangle_{\mathrm{oct}}=\langle\bar{s}\rangle_{\mathrm{oct}}, \quad\langle s\rangle_{\mathrm{dec}}=\langle\bar{s}\rangle_{\mathrm{dec}}, \quad\langle s\rangle_{\mathrm{tad}}=\langle\bar{s}\rangle_{\mathrm{bub}}$.

Although the contribution to the total strange and antiquark quark number from the decuplet intermediate states is about an order of magnitude smaller than that from octet intermediate states, the role of the kaon bubble and tadpole terms is more significant, making up $\approx 60 \%$ of the total. For the antistrange moment, $\langle\bar{s}\rangle$, including the $\delta$ function contributions from the rainbow diagrams, some $40 \%$ of the total moment comes from $x=0$. For the strange $\langle s\rangle$ moment, on the other hand, the structure of the convolution in Eq. (58) means that all of the contributions to $s(x)$ are at $x>0$, including ones involving $\delta$-function splitting functions. Interestingly, significant cancellation occurs between the local terms and the gauge linkdependent nonlocal contributions, which turn out to be negative and about half as large in magnitude as the local.

While the lowest moments of the $s$ and $\bar{s}$ are constrained to be equal, there is no such requirement for higher moments, including the $x$-weighted moment corresponding to the momentum carried by $s$ and $\bar{s}$ quarks. Since the total $s-\bar{s}$ asymmetry is found to be mostly positive over the range of $x$ relevant in this analysis, not surprisingly the total $\langle x(s-\bar{s})\rangle$ moment is also positive. Including the uncertainties on the kaon-nucleon-hyperon vertex regulator parameters from Fig. 6, the combined asymmetry in our analysis is

$$
\langle x(s-\bar{s})\rangle=\left(1.66_{-0.74}^{+0.81}\right) \times 10^{-3} .
$$

It is instructive, however, to observe the origin of the asymmetry in our chiral effective theory formulation. As mentioned above, there are no contributions to the momentum carried by $\bar{s}$ quarks from any of the $\delta$-function terms from the rainbow or kaon bubble diagrams, so that only the on-shell and on-shell end-point terms are nonzero. In contrast, all terms, including the $\delta$ function, contribute to the momentum carried by $s$ quarks. The result is a relatively small asymmetry that survives the cancellation of the (positive) on-shell $s$ and $\bar{s}$ terms, with large contributions 
TABLE VIII. Contributions from octet $Y=\Lambda, \Sigma^{0}, \Sigma^{+}$and decuplet $Y^{*}=\Sigma^{* 0}, \Sigma^{*+}$ hyperons to the average number (in units of $10^{-2}$ ) and momentum carried (in units of $10^{-3}$ ) by $s$ and $\bar{s}$ quarks in the nucleon from diagrams in Figs. 1 and 2 , for dipole regulator mass parameters $\Lambda_{K Y}=1.1(1) \mathrm{GeV}$ and $\Lambda_{K Y^{*}}=0.8(1) \mathrm{GeV}$. Note that some of the numbers do not sum to the totals because of rounding.

\begin{tabular}{|c|c|c|c|c|c|c|c|}
\hline & & $\langle\bar{s}\rangle\left(\times 10^{-2}\right)$ & $\langle x \bar{s}\rangle\left(\times 10^{-3}\right)$ & & & $\langle s\rangle\left(\times 10^{-2}\right)$ & $\langle x s\rangle\left(\times 10^{-3}\right)$ \\
\hline \multirow[t]{8}{*}{$K Y(\mathrm{rbw})$} & $f_{Y}^{(\text {on })}$ & $1.39_{-0.54}^{+0.69}$ & $1.33_{-0.56}^{+0.74}$ & $Y K($ rbw $)$ & $f_{Y}^{(\text {on })}$ & $1.39_{-0.54}^{+0.69}$ & $1.67_{-0.63}^{+0.78}$ \\
\hline & $f_{K}^{(\delta)}$ & $-\left(1.66_{-0.63}^{+0.79}\right)$ & 0 & & $f_{Y}^{(\text {off })}$ & $-\left(4.01_{-1.42}^{+1.68}\right)$ & $-\left(5.35_{-1.83}^{+2.12}\right)$ \\
\hline & $\delta f_{K}^{(\delta)}$ & $1.12_{-0.41}^{+0.50}$ & 0 & & $\delta f_{Y}^{(\text {off })}$ & $2.70_{-0.92}^{+1.07}$ & $3.12_{-1.02}^{+1.13}$ \\
\hline & & & & & $f_{K}^{(\delta)}$ & $1.66_{-0.63}^{+0.79}$ & $2.82_{-1.07}^{+1.35}$ \\
\hline & & & & $Y K(\mathrm{KR})$ & $f_{Y}^{(\mathrm{off})}$ & $4.01_{-1.42}^{+1.68}$ & $6.29_{-2.15}^{+2.50}$ \\
\hline & & & & & $f_{K}^{(\delta)}$ & $-\left(3.31_{-1.26}^{+1.58}\right)$ & $-\left(6.66_{-2.53}^{+3.18}\right)$ \\
\hline & & & & $Y K(\delta \mathrm{KR})$ & $\delta f_{Y}^{(\text {off })}$ & $-\left(2.70_{-0.92}^{+1.07}\right)$ & $-\left(3.68_{-1.20}^{+1.33}\right)$ \\
\hline & & & & & $\delta f_{K}^{(\delta)}$ & $1.12_{-0.41}^{+0.50}$ & $2.24_{-0.82}^{+1.01}$ \\
\hline Total octet & & $0.85_{-0.32}^{+0.40}$ & $1.33_{-0.56}^{+0.74}$ & Total octet & & $0.85_{-0.32}^{+0.40}$ & $0.46_{-0.14}^{+0.14}$ \\
\hline \multirow[t]{2}{*}{$K($ bub $)$} & $f_{K}^{(\delta)}$ & $4.85_{-1.84}^{+2.32}$ & 0 & $K(\operatorname{tad})$ & $f_{K}^{(\delta)}$ & $4.85_{-1.84}^{+2.32}$ & $7.87_{-2.98}^{+3.76}$ \\
\hline & $\delta f_{K}^{(\delta)}$ & $-\left(3.27_{-1.20}^{+1.47}\right)$ & 0 & $K(\delta \operatorname{tad})$ & $\delta f_{K}^{(\delta)}$ & $-\left(3.27_{-1.20}^{+1.47}\right)$ & $-\left(5.30_{-1.94}^{+2.38}\right)$ \\
\hline Total bubble & & $1.59_{-0.64}^{+0.85}$ & 0 & Total tadpole & & $1.59_{-0.64}^{+0.85}$ & $2.57_{-1.04}^{+1.38}$ \\
\hline \multirow[t]{16}{*}{$K Y^{*}(\mathrm{rbw})$} & $f_{Y^{*}}^{(\text {on })}$ & $0.09_{-0.07}^{+0.13}$ & $0.06_{-0.04}^{+0.09}$ & $Y^{*} K(\mathrm{rbw})$ & $f_{Y^{*}}^{(\text {on })}$ & $0.09_{-0.07}^{+0.13}$ & $0.10_{-0.08}^{+0.14}$ \\
\hline & $f_{Y^{*}}^{(\text {on end })}$ & $0.04_{-0.03}^{+0.07}$ & $0.03_{-0.03}^{+0.06}$ & & $f_{Y^{*}}^{(\text {on end })}$ & $0.04_{-0.03}^{+0.07}$ & $0.04_{-0.03}^{+0.07}$ \\
\hline & $f_{Y^{*}}^{(\delta)}$ & $-\left(0.01_{-0.01}^{+0.01}\right)$ & 0 & & $f_{Y^{*}}^{(\mathrm{off})}$ & $-\left(0.59_{-0.42}^{+0.72}\right)$ & $-\left(0.75_{-0.52}^{+0.89}\right)$ \\
\hline & $f_{K}^{(\delta)}$ & $-\left(0.15_{-0.11}^{+0.20}\right)$ & 0 & & $f_{Y^{*}}^{(\text {off end })}$ & $0.17_{-0.12}^{+0.23}$ & $0.21_{-0.15}^{+0.29}$ \\
\hline & $\delta f_{K}^{(\delta)}$ & $0.11_{-0.08}^{+0.14}$ & 0 & & $\delta f_{Y^{*}}^{(\mathrm{off})}$ & $0.34_{-0.24}^{+0.45}$ & $0.38_{-0.27}^{+0.47}$ \\
\hline & & & & & $f_{K}^{(\delta)}$ & $0.18_{-0.13}^{+0.24}$ & $0.26_{-0.19}^{+0.34}$ \\
\hline & & & & & $f_{Y^{*}}^{(\delta)}$ & $0.01_{-0.01}^{+0.01}$ & $0.01_{-0.01}^{+0.02}$ \\
\hline & & & & & $\delta f_{Y^{*}}^{(\delta)}$ & $-\left(0.07_{-0.05}^{+0.11}\right)$ & $-\left(0.10_{-0.07}^{+0.16}\right)$ \\
\hline & & & & $Y^{*} K(\mathrm{KR})$ & $f_{Y^{*}}^{(\mathrm{off})}$ & $0.59_{-0.42}^{+0.72}$ & $1.02_{-0.71}^{+1.21}$ \\
\hline & & & & & $f_{Y^{*}}^{(\text {off end })}$ & $-\left(0.17_{-0.12}^{+0.23}\right)$ & $-\left(0.29_{-0.21}^{+0.39}\right)$ \\
\hline & & & & & & $-\left(0.34_{-0.24}^{+0.44}\right)$ & $-\left(0.65_{-0.47}^{+0.85}\right)$ \\
\hline & & & & & & $-\left(0.02_{-0.01}^{+0.03}\right)$ & $-\left(0.03_{-0.02}^{+0.05}\right)$ \\
\hline & & & & & $\delta f_{Y^{*}}^{(\delta)}$ & $0.05_{-0.03}^{+0.08}$ & $0.09_{-0.07}^{+0.15}$ \\
\hline & & & & $Y^{*} K(\delta \mathrm{KR})$ & $\delta f_{Y^{*}}^{(\text {off })}$ & $-\left(0.34_{-0.24}^{+0.45}\right)$ & $-\left(0.51_{-0.36}^{+0.63}\right)$ \\
\hline & & & & & $\delta f_{K}^{(\delta)}$ & $0.11_{-0.08}^{+0.14}$ & $0.22_{-0.15}^{+0.27}$ \\
\hline & & & & & $\delta f_{Y^{*}}^{(\delta)}$ & $0.02_{-0.02}^{+0.04}$ & $0.05_{-0.03}^{+0.07}$ \\
\hline Total decuplet & & $0.08_{-0.06}^{+0.12}$ & $0.09_{-0.07}^{+0.15}$ & Total decuplet & & $0.08_{-0.06}^{+0.12}$ & $0.04_{-0.03}^{+0.04}$ \\
\hline Total & & $2.51_{-1.02}^{+1.36}$ & $1.42_{-0.62}^{+0.89}$ & Total & & $2.51_{-1.02}^{+1.36}$ & $3.08_{-1.20}^{+1.55}$ \\
\hline Non- $\delta$ function & & $1.52_{-0.64}^{+0.88}$ & $1.42_{-0.62}^{+0.89}$ & Non- $\delta$ function & & $1.52_{-0.64}^{+0.88}$ & $2.25_{-0.92}^{+1.21}$ \\
\hline$\delta$ function & & $0.99_{-0.50}^{+0.60}$ & 0 & $\delta$ function & & $0.99_{-0.50}^{+0.60}$ & $0.82_{-0.63}^{+0.67}$ \\
\hline Local & & $4.55_{-1.77}^{+2.23}$ & $1.42_{-0.62}^{+0.89}$ & Local & & $4.55_{-1.77}^{+2.23}$ & $6.58_{-2.60}^{+3.14}$ \\
\hline Nonlocal & & $-\left(2.04_{-0.92}^{+1.04}\right)$ & 0 & Nonlocal & & $-\left(2.04_{-0.92}^{+1.04}\right)$ & $-\left(3.50_{-1.46}^{+1.66}\right)$ \\
\hline
\end{tabular}



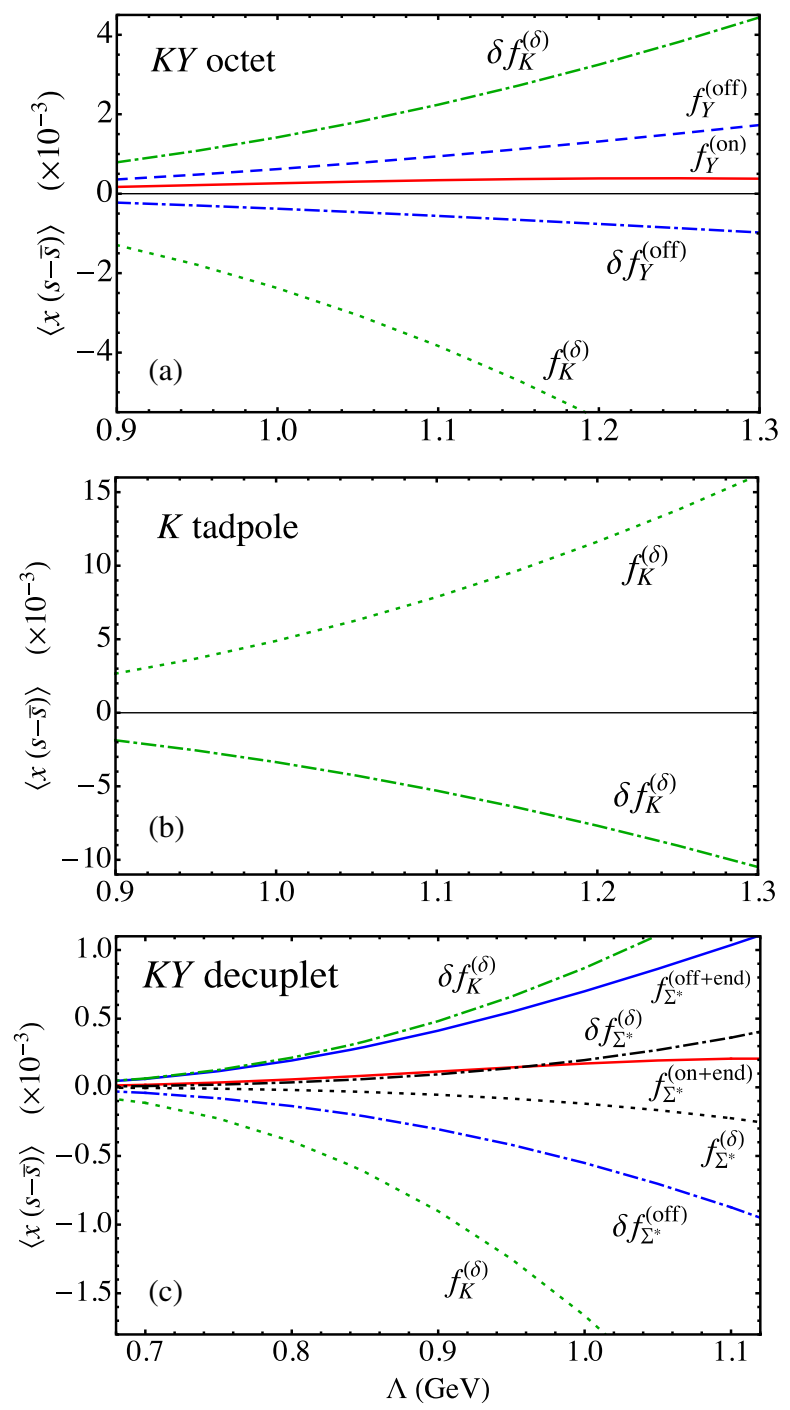

FIG. 8. Contributions to the $\langle x(s-\bar{s})\rangle$ moment vs the dipole cutoff parameter $\Lambda\left(=\Lambda_{K Y}\right.$, for $Y=\Lambda$ or $\Sigma$ hyperons, or $\left.\Lambda_{K \Sigma^{*}}\right)$ from (a) the $K Y$ octet rainbow [Figs. 1(a) and 2(d)] and KrollRuderman [Figs. 2(e)-2(f)] diagrams; (b) the $K$ bubble [Fig. 1(c)] and tadpole [Figs. 2(j) and 2(k)] diagrams; (c) the $K \Sigma^{*}$ decuplet rainbow [Figs. 1(b) and 2(g)] and Kroll-Ruderman [Fig. 2(h) and 2(i)] diagrams.

from individual off-shell and $\delta$-function terms. As illustrated in Fig. (8) for the various contributions to the strange momentum asymmetry vs the regulator cutoff mass, the largest of these in magnitude is the (negative) $f_{K}^{(\delta)}$ term from the Kroll-Ruderman diagram [Fig. 2(e)], with comparably large (positive) gauge link contributions $\delta f_{K}^{(\delta)}$ from the rainbow [Fig. 2(d)] and nonlocal Kroll-Ruderman [Fig. 2(f)] diagrams. After the cancellations of various terms, the octet baryon contribution to the strange momentum asymmetry is actually negative, $\langle x(s-\bar{s})\rangle_{\text {oct }} \approx-0.87 \times 10^{-3}$. The terms involving decuplet hyperon states give relatively small absolute contributions, with significant cancellations arising that lead to a negligible overall strange decuplet asymmetry, $\langle x(s-\bar{s})\rangle_{\mathrm{dec}} \approx-0.05 \times 10^{-3}$.

Interestingly, the most significant role played here is by the kaon tadpole terms [Figs. 2(j) and 2(k)]. With strong cancellations between the positive local $f_{K}^{(\delta)}$ and negative nonlocal $\delta f_{K}^{(\delta)}$ terms, the total asymmetry from the tadpole, $\langle x(s-\bar{s})\rangle_{\mathrm{tad}} \approx 2.57 \times 10^{-3}$, is still about 3 times larger in magnitude than that from the rainbow and Kroll-Ruderman diagrams. The result is an overall asymmetry in Eq. (73) that is positive.

Experimentally, identifying an asymmetry of this size will be challenging, but not impossible. Traditionally, inclusive charm meson production in charged current neutrino and antineutrino DIS from nuclei has been used to provide information about the $s$ and $\bar{s}$ PDFs in the nucleon, and analyses of data from neutrino experiments at BEBC [60], CDHS [61], CDHSW [62], CCFR [63], and $\mathrm{NuTeV}[64,65]$ have yielded values in the range $\langle x(s-\bar{s})\rangle \sim(0-3) \times 10^{-3}$ [66-68]. Unfortunately, the neutrino-nucleus data are known to be affected by uncertainties in nuclear medium effects when relating nuclear structure functions to those of free nucleons [69], and in the nuclear dependence of charm quark energy loss and $D$ meson interactions during hadronization in the nuclear medium [70,71].

Alternatively, the $s$ and $\bar{s}$ distributions can be constrained by $K^{ \pm}$meson production data from semiinclusive deep-inelastic scattering (SIDIS) off protons and deuterons, such as from the HERMES [72,73] or COMPASS [74] experiments. In a first of its kind global analysis, the JAM Collaboration recently fitted both the SIDIS and inclusive DIS data, along with other high energy scattering data, within a Bayesian likelihood analysis using Monte Carlo techniques to simultaneously determine both the spin-averaged PDFs and parton-tohadron fragmentation functions [75]. The analysis found a suppressed strange content in the nucleon at large $x$, and found no clear evidence for a nonzero $s-\bar{s}$ asymmetry within relatively large uncertainties. In the future, high-precision SIDIS data from the Jefferson Lab $12 \mathrm{GeV}$ program or from the planned Electron-Ion Collider should provide better constraints on the $s$ and $\bar{s}$ PDFs, as may $W+$ charm production data from $p p$ collisions at the LHC [76-78].

An important consequence of a better determination of the $s-\bar{s}$ asymmetry in the nucleon is more robust constraints on the weak mixing angle $\sin ^{2} \theta_{W}$ extracted from the NuTeV data on $\nu$ and $\bar{\nu}$ nuclear cross sections $[68,79$ 81]. For the total strange asymmetries range found in this analysis, $0.9 \times 10^{-3} \lesssim\langle x(s-\bar{s})\rangle \lesssim 2.5 \times 10^{-3}$, the resulting correction to the weak angle lies in the range $-2.4 \times 10^{-3} \lesssim \Delta \sin ^{2} \theta_{W} \lesssim-0.9 \times 10^{-3}$, or between $18 \%$ and $49 \%$ of the total quoted discrepancy [64,79] (see Ref. [82] for a review and further discussion). 


\section{CONCLUSION}

In this paper we have calculated the contributions to the sea quark distributions in the proton which are generated within a nonlocal chiral effective field theory. Both octet and decuplet intermediate states were included in the oneloop calculation using a four-dimensional dipole regulator to deal with the ultraviolet divergences. This regulator was introduced explicitly in the nonlocal Lagrangian density, with gauge invariance ensured through the presence of gauge links. A consequence of the introduction of the regulator are additional diagrams [Figs. 2(f), 2(i), and 2(k)] that arise from the expansion of the gauge links to lowest order in the electromagnetic coupling.

The free parameters entering the calculation, namely the dipole regulator masses, have been determined by fitting the available inclusive differential $p p \rightarrow n X$, $p p \rightarrow \Delta X, p p \rightarrow \Lambda X$, and $p p \rightarrow \Sigma^{*} X$ cross section data. Using the fitted values of the dipole masses, $\Lambda=$ $\{1.0(1), 0.9(1), 1.1(1), 0.8(1)\} \mathrm{GeV}$ for the $\{\pi N, \pi \Delta$, $\left.K \Lambda, K \Sigma^{*}\right\}$ states, respectively, we computed the $x$ dependence of the sea quark asymmetry $\bar{d}-\bar{u}$, which is dominated at $x>0$ by the on-shell contribution involving a nucleon intermediate state. The general shape and magnitude of the asymmetry extracted from the E866 Drell-Yan data [4] are described quite well, with the exception of the apparent change sign at higher $x$ values, which is practically impossible to accommodate within the current theoretical framework. On the other hand, preliminary data from the SeaQuest experiment at Fermilab [83] suggest that the extracted $\bar{d} / \bar{u}$ ratio may flatten out at large $x$ values and remain above unity. The integrated $\bar{d}-\bar{u}$ asymmetry was found to lie in the range between $\langle\bar{d}-\bar{u}\rangle \approx 0.09$ and 0.17 , which encompasses the values extracted by the New Muon [1] and E866 [4] Collaborations of $\approx 0.15$ and 0.12, respectively. Remarkably, some $30 \%$ of our calculated value is associated with a $\delta$-function contribution at $x=0$, which is not accessible experimentally at finite energy.

For the strange distributions in the proton, both the $s$ and $\bar{s}$ PDFs were found to be positive at all values of $x>0$. Interestingly in this case, while the $\bar{s}$ distribution receives $\delta$-function contributions also at $x=0$ (around 2/3 of the total), the $s$ PDF vanishes at $x=0$; both integrate to the same value, however, to ensure zero total strangeness, $\langle s\rangle=\langle\bar{s}\rangle$. Again, the contributions from the octet baryon intermediate states are dominant, with decuplet baryon contributions about an order of magnitude smaller. The gauge link dependent terms play a significant role in the nonlocal formulation of the chiral theory, contributing about half of the total $\langle s\rangle$ and $\langle\bar{s}\rangle$, but of opposite sign.

Large cancellations also appear in the $x$-weighted asymmetry $x(s-\bar{s})$, which remains small but positive across all $x$, with the integrated value lying in the range $0.9 \times 10^{-3} \lesssim\langle x(s-\bar{s})\rangle \lesssim 2.5 \times 10^{-3}$. This is broadly consistent with previous determinations from neutrino scattering experiments $[63,65]$, although the uncertainties on the empirical bounds are rather large. A nonzero moment $\langle x(s-\bar{s})\rangle$ leads to a correction [68] to the NuTeV extraction of $\sin ^{2} \theta_{W}$ [79]. Our result supports the idea that the strange-antistrange quark asymmetry may indeed reduce the $\mathrm{NuTeV}$ anomaly by up to 1 standard deviation, which, along with other corrections such as charge symmetry breaking in the nucleon sea [84-86] and the isovector EMC effect [87], may account for the apparent anomaly entirely in terms of Standard Model physics.

Future progress on constraining the $s-\bar{s}$ asymmetry experimentally is expected to come on several fronts. Parity-violating inclusive DIS and semi-inclusive kaon electroproduction from hydrogen at Jefferson Lab and at a future Electron-Ion Collider will provide independent combinations of flavor PDFs, with the $s$ and $\bar{s}$ distributions weighted by different electroweak charges and fragmentation functions, respectively. At higher energies, data on inclusive $W+$ charm production in $p p$ collisions at the LHC $[76,77]$ can also provide sensitivity to differences between the $s$ and $\bar{s}$ PDFs at small values of $x$, complementing the constraints at higher $x$ values from fixed target experiments.

\section{ACKNOWLEDGMENTS}

This work is supported by NSFC under Grant No. 11475186, the Sino-German CRC 110 "Symmetries and the Emergence of Structure in QCD" project by NSFC under the Grant No. 11621131001, the DOE Contract No. DE-AC05-06OR23177, under which Jefferson Science Associates, LLC operates Jefferson Lab, DOE Contract No. DE-FG02-03ER41260, and by the Australian Research Council through the ARC Centre of Excellence for Particle Physics at the Terascale (CE110001104) and Discovery Projects No. DP151103101 and No. DP180100497.
[1] M. Arneodo et al., Phys. Rev. D 50, R1 (1994).

[2] K. Ackerstaff et al., Phys. Rev. Lett. 81, 5519 (1998).

[3] A. Baldit et al., Phys. Lett. B 332, 244 (1994).

[4] R. S. Towell et al., Phys. Rev. D 64, 052002 (2001).
[5] A. W. Thomas, Phys. Lett. 126B, 97 (1983).

[6] A. W. Thomas, Adv. Nucl. Phys. 13, 1 (1984).

[7] A. W. Thomas, S. Theberge, and G. A. Miller, Phys. Rev. D 24, 216 (1981). 
[8] D. F. Geesaman and P. E. Reimer, Rep. Prog. Phys. 82, 046301 (2019).

[9] A. Szczurek, J. Speth, and G. T. Garvey, Nucl. Phys. A570, 765 (1994).

[10] J. Magnin and H. R. Christiansen, Phys. Rev. D 61, 054006 (2000).

[11] J. Ashman et al., Nucl. Phys. B328, 1 (1989).

[12] J. R. Ellis and R. L. Jaffe, Phys. Rev. D 9, 1444 (1974).

[13] S. Catani, D. de Florian, G. Rodrigo, and W. Vogelsang, Phys. Rev. Lett. 93, 152003 (2004).

[14] A. I. Signal and A. W. Thomas, Phys. Lett. B 191, 205 (1987).

[15] W. Melnitchouk and M. Malheiro, Phys. Rev. C 55, 431 (1997).

[16] R. S. Sufian, T. Liu, G. F. de Téramond, H. G. Dosch, S. J. Brodsky, A. Deur, M. T. Islam, and B.-Q. Ma, Phys. Rev. D 98, 114004 (2018).

[17] L. A. Trevisan and C. Mirez, Int. J. Mod. Phys. A 33, 1850083 (2018).

[18] A. Vega, I. Schmidt, T. Gutsche, and V. E. Lyubovitskij, Phys. Rev. D 93, 056001 (2016).

[19] G. Q. Feng, F. G. Cao, X. H. Guo, and A. I. Signal, Eur. Phys. J. C 72, 2250 (2012).

[20] Y. Salamu, C.-R. Ji, W. Melnitchouk, A. W. Thomas, and P. Wang, Phys. Rev. D 99, 014041 (2019).

[21] F. He and P. Wang, Phys. Rev. D 97, 036007 (2018).

[22] F. He and P. Wang, Phys. Rev. D 98, 036007 (2018).

[23] J.-W. Chen and X. Ji, Phys. Rev. Lett. 87, 152002 (2001); 88, 249901(E) (2002).

[24] X. G. Wang, C.-R. Ji, W. Melnitchouk, Y. Salamu, A. W. Thomas, and P. Wang, Phys. Rev. D 94, 094035 (2016).

[25] X. G. Wang, C. R. Ji, W. Melnitchouk, Y. Salamu, A. W. Thomas, and P. Wang, Phys. Lett. B 762, 52 (2016).

[26] C. R. Ji, W. Melnitchouk, and A. W. Thomas, Phys. Rev. D 88, 076005 (2013).

[27] P. E. Shanahan, A. W. Thomas, and R. D. Young, Phys. Rev. D 87, 114515 (2013).

[28] C. Hacker, N. Wies, J. Gegelia, and S. Scherer, Phys. Rev. C 72, 055203 (2005).

[29] L. M. Nath, B. Etemadi, and J. D. Kimel, Phys. Rev. D 3, 2153 (1971).

[30] A. M. Moiseeva and A. A. Vladimirov, Eur. Phys. J. A 49, 23 (2013).

[31] J. N. Labrenz and S. R. Sharpe, Phys. Rev. D 54, 4595 (1996).

[32] X. G. Wang et al. (to be published).

[33] E. E. Jenkins, Nucl. Phys. B368, 190 (1992).

[34] M. Burkardt, K. S. Hendricks, C.-R. Ji, W. Melnitchouk, and A. W. Thomas, Phys. Rev. D 87, 056009 (2013).

[35] Y. Salamu, C.-R. Ji, W. Melnitchouk, and P. Wang, Phys. Rev. Lett. 114, 122001 (2015).

[36] H. Holtmann, A. Szczurek, and J. Speth, Nucl. Phys. A596, 631 (1996).

[37] W. Melnitchouk, J. Speth, and A. W. Thomas, Phys. Rev. D 59, 014033 (1998).

[38] W. Flauger and F. Monnig, Nucl. Phys. B109, 347 (1976).

[39] V. Blobel, H. Fesefeldt, H. Franz, W. M. Geist, K. Von Holt, U. Idschok, and N. Schmitz, Nucl. Phys. B135, 379 (1978).
[40] S. J. Barish, M. Derrick, B. Musgrave, P. Schultz, J. Whitmore, R. Engelmann, T. Kafka, M. Pratap, and R. D. Field, Phys. Rev. D 12, 1260 (1975).

[41] K. Jaeger, J. Campbell, G. Charlton, D. Swanson, C. Fu, H. A. Rubin, R. G. Glasser, D. Koetke, and J. Whitmore, Phys. Rev. D 11, 1756 (1975).

[42] K. Bockmann, J. Hofmann, U. Idschok, B. Schröder, V. Blobel, H. Fesefeldt, and H. Franz, Nucl. Phys. B143, 395 (1978).

[43] M. Aicher, A. Schäfer, and W. Vogelsang, Phys. Rev. Lett. 105, 252003 (2010).

[44] A. D. Martin, R. G. Roberts, W. J. Stirling, and R. S. Thorne, Eur. Phys. J. C 4, 463 (1998).

[45] E. Leader, A. V. Sidorov, and D. B. Stamenov, Phys. Rev. D 82, 114018 (2010).

[46] P. C. Barry, N. Sato, W. Melnitchouk, and C. R. Ji, Phys. Rev. Lett. 121, 152001 (2018).

[47] J. F. Owens, Phys. Rev. D 30, 943 (1984).

[48] P. J. Sutton, A. D. Martin, R. G. Roberts, and W. J. Stirling, Phys. Rev. D 45, 2349 (1992).

[49] M. Glück, E. Reya, and A. Vogt, Z. Phys. C 53, 651 (1992).

[50] K. Wijesooriya, P. E. Reimer, and R. J. Holt, Phys. Rev. C 72, 065203 (2005).

[51] P. Jimenez-Delgado, W. Melnitchouk, and J.F. Owens, J. Phys. G 40, 093102 (2013).

[52] S. Forte and G. Watt, Annu. Rev. Nucl. Part. Sci. 63, 291 (2013).

[53] J. Hüfner and B. Povh, Phys. Rev. D 46, 990 (1992).

[54] J. R. McKenney, N. Sato, W. Melnitchouk, and C. R. Ji, Phys. Rev. D 93, 054011 (2016).

[55] W. Melnitchouk and A. W. Thomas, Phys. Rev. D 47, 3794 (1993).

[56] B. Z. Kopeliovich, I. K. Potashnikova, B. Povh, and I. Schmidt, Phys. Rev. D 85, 114025 (2012).

[57] U. D'Alesio and H. J. Pirner, Eur. Phys. J. A 7, 109 (2000).

[58] C. Adloff et al., Nucl. Phys. B619, 3 (2001).

[59] A. S. Carroll et al., Phys. Lett. 80B, 423 (1979).

[60] G. T. Jones et al., Z. Phys. C 62, 575 (1994).

[61] H. Abramowicz et al., Z. Phys. C 25, 29 (1984).

[62] P. Berge et al., Z. Phys. C 49, 187 (1991).

[63] A. O. Bazarko et al., Z. Phys. C 65, 189 (1995).

[64] G. P. Zeller et al., Phys. Rev. D 65, 111103(R) (2002); 67, 119902(E) (2003).

[65] D. Mason et al., Phys. Rev. Lett. 99, 192001 (2007).

[66] V. Barone, C. Pascaud, and F. Zomer, Eur. Phys. J. C 12, 243 (2000).

[67] S. Davidson, S. Forte, P. Gambino, N. Rius, and A. Strumia, J. High Energy Phys. 02 (2002) 037.

[68] W. Bentz, I. C. Cloët, J. T. Londergan, and A. W. Thomas, Phys. Lett. B 693, 462 (2010).

[69] N. Kalantarians, C. Keppel, and M. E. Christy, Phys. Rev. C 96, 032201 (2017).

[70] A. Accardi, F. Arleo, W. K. Brooks, D. D'Enterria, and V. Muccifora, Riv. Nuovo Cimento 32, 439 (2010).

[71] A. Majumder and M. Van Leeuwen, Prog. Part. Nucl. Phys. A 66, 41 (2011).

[72] A. Airapetian et al., Phys. Lett. B 666, 446 (2008).

[73] A. Airapetian et al., Phys. Rev. D 89, 097101 (2014). 
[74] C. Adolph et al., Phys. Lett. B 767, 133 (2017).

[75] N. Sato, C. Andres, J. J. Ethier, and W. Melnitchouk, arXiv: 1905.03788.

[76] G. Aad et al., J. High Energy Phys. 05 (2014) 068.

[77] S. Chatrchyan et al., J. High Energy Phys. 02 (2014) 013.

[78] A. M. Cooper-Sarkar and K. Wichmann, Phys. Rev. D 98, 014027 (2018).

[79] G. P. Zeller et al., Phys. Rev. Lett. 88, 091802 (2002); 90, 239902(E) (2003).

[80] F. X. Wei and B. S. Zou, Phys. Lett. B 660, 501 (2008).

[81] B. Q. Ma, Int. J. Mod. Phys. A 21, 930 (2006).

[82] J. T. Londergan, Nucl. Phys. B, Proc. Suppl. 141, 68 (2005).
[83] A. Tadepalli, Recent progress on studies of light quark flavor asymmetry at SeaQuest experiment, APS April Meeting, Denver, Colorado (2019), http://meetings.aps .org/Meeting/APR19/Session/X09.7.

[84] E. N. Rodionov, A. W. Thomas, and J. T. Londergan, Mod. Phys. Lett. A 09, 1799 (1994).

[85] J. T. Londergan and A. W. Thomas, Phys. Rev. D 67, 111901 (2003).

[86] J. T. Londergan, J.-C. Peng, and A. W. Thomas, Rev. Mod. Phys. 82, 2009 (2010).

[87] I. C. Cloët, W. Bentz, and A. W. Thomas, Phys. Rev. Lett. 102, 252301 (2009). 